\title{
POINCARÉ INDEX AND PERIODIC SOLUTIONS OF PERTURBED AUTONOMOUS SYSTEMS
}

\author{
O. YU. MAKARENKOV
}

\begin{abstract}
Classical conditions for the bifurcation of periodic solutions in perturbed auto-oscillating and conservative systems go back to Malkin and Mel'nikov, respectively. These authors' papers were based on the Lyapunov-Schmidt reduction and the implicit function theorem, which lead to the requirement that both the cycles and the zeros of the bifurcation functions be simple. In this paper a geometric approach is put forward which does not assume these requirements, but imposes a certain condition on the Poincaré index of a generating cycle with respect to some auxiliary vector field. The approach is based on calculating the topological degree of the Poincaré operator of the perturbed system with respect to interior and exterior neighbourhoods of a generating cycle, as a consequence of which the conclusion of the main theorem guarantees bifurcation of a certain number of periodic solutions towards the interior of the cycle, and of a certain number of periodic solutions towards the exterior of the cycle. Concrete examples are given, where this approach either establishes bifurcation of a greater number of periodic solutions compared with the known classical results, or provides additional information on the location of these solutions.
\end{abstract}

\section{INTRODUCTION}

We consider an autonomous system of ordinary differential equations

$$
\dot{x}=f(x), \quad x \in \mathbb{R}^{2},
$$

where $f$ is a continuously differentiable function such that a solution of the system (1.1) with any initial condition can be extended to the interval $(-\infty, \infty)$. We call the system (1.1) a generating system. Suppose that the system (1.1) admits a $T$-periodic cycle $\widetilde{x}$. Since the system is autonomous, for any shift $\theta \in[0, T]$ the function

$$
\widetilde{x}^{\theta}(t)=\widetilde{x}(\theta+t)
$$

is again a $T$-periodic solution of the system (1.1). Suppose that a function $g: \mathbb{R} \times \mathbb{R}^{2} \rightarrow \mathbb{R}^{2}$ is $T$-periodic in the first variable and consider the perturbed system

$$
\dot{x}=f(x)+\varepsilon g(t, x), \quad x \in \mathbb{R}^{2} .
$$

The paper is devoted to the classical problem, which goes back to Poincaré, of whether the perturbed system (1.2) has $T$-periodic solutions $\widetilde{x}_{\varepsilon}$ which converge to a generating solution $\widetilde{x}^{\theta}$ as $\varepsilon \rightarrow 0$. Poincaré's assertion states that the parameter $\theta=\theta_{0}$ of the

2000 Mathematics Subject Classification. Primary 34C25; Secondary 34A26, 34C23, 34D10, 47H11.

Key words and phrases. Poincaré index, periodic solution, bifurcation, perturbation.

This research was supported by the Russian Federal Agency for Science and Innovation and the Programme for Basic Research and Higher Education of the U.S. Civilian Research and Development Foundation (grant BF6M10), by the President of the Russian Federation fund for the support of young scientists (grant MK-1620.2008.1) and by the Marie Curie Foundation (grant PIIF-GA-2008-221331).

The main results of the paper were reported at the seminar of the Department of Differential Equations of the Steklov Mathematical Institute of the Russian Academy of Sciences on 22 March, 2006. 
generating solution for which such solutions exist is necessarily a zero of some bifurcation function $M$. Poincaré wrote the function $M$ in implicit form.

Later Malkin [24] and Mel'nikov [27. found an explicit form for the function $M$ and by using the implicit function theorem proved that, in various situations, a sufficient condition for the system (1.2) to have a $T$-periodic solution $\widetilde{x}_{\varepsilon}$ converging to $\widetilde{x}^{\theta_{0}}$ as $\varepsilon \rightarrow 0$ is given by the condition

$$
M^{\prime}\left(\theta_{0}\right) \neq 0
$$

that is, the root $\theta_{0}$ must be simple.

The difference between the situations studied by Malkin and Mel'nikov is related to conditions imposed on the $T$-periodic linear system

$$
\dot{y}=f^{\prime}(\widetilde{x}(t)) y .
$$

For example, Malkin assumed that

$$
\begin{aligned}
\left(C_{M A}\right): & \text { the algebraic multiplicity of the multiplicator }+1 \\
& \text { of the system (1.4) is equal to } 1,
\end{aligned}
$$

whilst Mel'nikov assumed that

$$
\begin{aligned}
\left(C_{M E}\right): & \text { the algebraic multiplicity of the multiplicator }+1 \\
& \text { of the system (1.4) is equal to } 2, \\
& \text { but the geometric multiplicity of the multiplicator }+1 \\
& \text { of the system (1.4) is equal to } 1 .
\end{aligned}
$$

In this paper we show that another of Poincaré's ideas, concerning the index of a curve with respect to a vector field, lets us obtain similar results under only one assumption:

$$
\begin{aligned}
(C): & \text { in a small neighbourhood of the cycle } \widetilde{x} \text { there are no other } \\
& T \text {-periodic solutions of the generating system. }
\end{aligned}
$$

Condition $(C)$ holds both in Malkin's situation $\left(C_{M A}\right)$ and in Mel'nikov's situation $\left(C_{M E}\right)$. But the assumption $(C)$ does not use any properties of the linearized system (1.4) and, consequently, can be satisfied for degenerate cases, where each solution of the system (1.4) is $T$-periodic; see $\S 5$.

By contrast with the results due to Malkin and Mel'nikov, this approach does not assume that the perturbation is differentiable; it is related to extensions of Poincaré index theory; see [16]. Although the main theorem is proved for the case of a continuous perturbation, by following standard schemes (see, for example, [12, §6.2]) it can be extended not only to the case of discontinuous perturbations but also multivalued perturbations with convex images.

We shall show that conditions for the existence of periodic solutions of the perturbed system near the cycle $\widetilde{x}$ can be related to the number of revolutions performed by the vector

$$
\Phi(\widetilde{x}(\theta))=M_{E}(\theta) \dot{\widetilde{x}}(\theta)^{\perp}+M_{A}(\theta) \dot{\tilde{x}}(\theta)
$$

when $\theta$ varies from 0 to $T$. Here we used the notation

$$
\xi^{\perp}=\left(\begin{array}{c}
-\xi_{2} \\
\xi_{1}
\end{array}\right) .
$$

The number of revolutions is called the Poincaré index of the cycle $\widetilde{x}$ with respect to the field $\Phi$ defined on the cycle $\widetilde{x}$ and is denoted by $\operatorname{ind}(\widetilde{x}, \Phi)$. Here we do not dwell on the definition of the Poincaré index, which can be found, for example, in 38, Ch. III, XIV], [21, Ch. IX, §4] and [2, Ch. V, § 10.4]. Since in this literature the Poincaré index is introduced for a positively oriented curve, in what follows we always assume that the 
curve $\widetilde{x}$ is positively oriented. The functions $M_{A}$ and $M_{E}$ of formula (1.5), which are defined in the next section, coincide with the classical functions of Malkin and Mel'nikov under certain additional conditions.

\section{The MAIN RESUlt}

To define the functions $M_{E}$ and $M_{A}$ we consider solutions of the following conjugate system:

$$
\dot{z}=-\left(f^{\prime}(\widetilde{x}(t))\right)^{*} z .
$$

Namely, we denote by $\widetilde{z}$ a solution of the system (2.6) with the initial condition

$$
\widetilde{z}(0)=\dot{\widetilde{x}}(0)^{\perp},
$$

and by $\widehat{z}$ a solution of the system (2.6) with the initial condition

$$
\widehat{z}(0)=\frac{1}{\|\dot{\widetilde{x}}(0)\|^{2}} \dot{\tilde{x}}(0) .
$$

Thus, we set

$$
\begin{aligned}
& M_{E}^{s}(\theta)=\int_{s-T+\theta}^{s+\theta}\langle\widetilde{z}(\tau), g(\tau-\theta, \widetilde{x}(\tau))\rangle d \tau, \\
& M_{A}^{s}(\theta)=\int_{s-T+\theta}^{s+\theta}\langle\widehat{z}(\tau), g(\tau-\theta, \widetilde{x}(\tau))\rangle d \tau, \quad \theta, s \in[0, T],
\end{aligned}
$$

where $\langle\cdot, \cdot\rangle$ is the ordinary inner product in $\mathbb{R}^{2}$. Furthermore, we assume by definition that

$$
M_{E}:=M_{E}^{0}, \quad M_{A}:=M_{A}^{0} .
$$

We will now state the main theorem.

Theorem 2.1. Suppose that condition $(C)$ holds and the perturbation in (1.2) is continuous. Suppose that

$$
\text { for any } s, \theta \in[0, T] \text {, the equality } M_{E}^{s}(\theta)=0 \text { implies } M_{A}^{s}(\theta) \neq 0 .
$$

Then for sufficiently small $\varepsilon>0$, every T-periodic solution $\widetilde{x}_{\varepsilon}$ of the perturbed system (1.2) is necessarily such that

$$
\widetilde{x}_{\varepsilon}(t) \neq \widetilde{x}(s) \quad \text { for all } t, s \in[0, T] .
$$

If in addition

$$
\operatorname{ind}(\widetilde{x}, \Phi) \neq 1
$$

then for all sufficiently small $\varepsilon>0$, the perturbed system (1.2) does have at least two $T$-periodic solutions $\widetilde{x}_{\varepsilon, 1}$ and $\widetilde{x}_{\varepsilon, 2}$ satisfying condition (2.7). To these solutions there correspond $\theta_{1}, \theta_{2} \in[0, T]$ such that

$$
\widetilde{x}_{\varepsilon, 1}(t) \rightarrow \widetilde{x}^{\theta_{1}}(t) \quad \text { as } \varepsilon \rightarrow 0
$$

and

$$
\widetilde{x}_{\varepsilon, 2}(t) \rightarrow \widetilde{x}^{\theta_{2}}(t) \quad \text { as } \varepsilon \rightarrow 0 .
$$

Moreover, the solution $\widetilde{x}_{\varepsilon, 1}$ is contained inside the cycle $\widetilde{x}$, and the solution $\widetilde{x}_{\varepsilon, 2}$ outside; that is, $\widetilde{x}_{\varepsilon, 1}([0, T]) \subset U$ and $\widetilde{x}_{\varepsilon, 2}([0, T]) \subset \mathbb{R}^{2} \backslash U$, where $U \subset \mathbb{R}^{2}$ is the open bounded set whose boundary is $\widetilde{x}([0, T])$. 
The existence of the open bounded set $U$ mentioned in Theorem 2.1 follows from Jordan's theorem (see [1, Ch. II]).

We will now proceed to prove Theorem 2.1. To do this, first we introduce some concepts we need and establish some auxiliary results.

We denote by $\Omega\left(\cdot, t_{0}, \xi\right)$ a solution $x$ of the generating system (1.1) with the initial condition $x\left(t_{0}\right)=\xi$. We consider the system

$$
\dot{y}=f_{(2)}^{\prime}(t, \Omega(t, 0, \xi)) y+g(t, \Omega(t, 0, \xi))
$$

(see [24, formula 3.8]) and denote by $\eta(\cdot, s, \xi)$ a solution $y$ of this system with the initial condition $y(s)=0$. Henceforth $F_{(k)}^{\prime}$ denotes the derivative of a function $F$ with respect to the $k$ th variable. We associate with the system (1.2) the following operator $\widetilde{\Phi}^{s}: \mathbb{R}^{2} \rightarrow \mathbb{R}^{2}$ (see [15, Theorem 1]):

$$
\widetilde{\Phi}^{s}(\xi)=\eta(T, s, \xi)-\eta(0, s, \xi) .
$$

We point out that the operator $\widetilde{\Phi}^{0}$ does not coincide with $\Phi$, but we will show later that the Poincaré indices of these operators calculated on the cycle $\widetilde{x}$ coincide. When $f=0$, that is, when the generating system is the system $\dot{x}=0$, we have

$$
\widetilde{\Phi}^{s}(\xi)=\int_{0}^{T} g(\tau, \xi) d \tau, \quad s \in \mathbb{R}, \xi \in \mathbb{R}^{2} .
$$

Consequently, if $f=0$, then $\widetilde{\Phi}^{s}$ is, up to the factor $1 / T$, the classical Krylov-BogolyubovMitropol'skiu averaging operator of the perturbed system (1.2) (see [28, formula 7.112]).

We denote by $\widehat{y}$ a solution of the linearized system (1.4) with the initial condition

$$
\widehat{y}(0)=\frac{1}{\|\dot{\widetilde{x}}(0)\|^{2}} \dot{\tilde{x}}(0)^{\perp} .
$$

Then we have the next result.

Lemma 2.1. The following formula holds:

$$
\widetilde{\Phi}^{s}(\widetilde{x}(\theta))=M_{A}^{s}(\theta) \dot{\widetilde{x}}(\theta)+M_{E}^{s}(\theta) \widehat{y}(\theta), \quad s, \theta \in \mathbb{R} .
$$

To prove this lemma, and in subsequent arguments, we will use the next result.

Lemma 2.2 (see [13, Lemma 1] or [14, Lemma 2]). The formula

$$
\eta(\theta, s, \xi)=\Omega_{(3)}^{\prime}(\theta, 0, \xi) \int_{s}^{\theta} \Omega_{(3)}^{\prime}(0, t, \Omega(t, 0, \xi)) g(t, \Omega(t, 0, \xi)) d \tau
$$

holds; correspondingly,

$$
\widetilde{\Phi}^{s}(\xi)=\int_{s-T}^{s} \Omega_{(3)}^{\prime}(0, \tau, \Omega(\tau, 0, \xi)) g(\tau, \Omega(\tau, 0, \xi)) d \tau .
$$

Proof of Lemma 2.1. By Lemma 2.2 we have

$$
\widetilde{\Phi}^{s}(\xi)=\int_{s-T}^{s}(\Omega)_{(3)}^{\prime}(0, \tau, \Omega(\tau, 0, \xi)) g(\tau, \Omega(\tau, 0, \xi)) d \tau .
$$

Next (see, for example, 17, Theorem 2.1]), $\Omega_{(3)}^{\prime}\left(t, 0, x_{0}(\theta)\right)=Y(t, \theta)$, where $Y(t, \theta)$ is a fundamental matrix of the system

$$
\dot{y}(t)=f^{\prime}(\widetilde{x}(t+\theta)) y(t)
$$

satisfying the condition $Y(0, \theta)=I$, and, since

$$
\Omega_{(3)}^{\prime}(0, t, \Omega(t, 0, \xi)) \Omega_{(3)}^{\prime}(t, 0, \xi)=I \quad \text { for any } t \in \mathbb{R}, \xi \in \mathbb{R}^{2},
$$


we have

$$
\widetilde{\Phi}^{s}(\widetilde{x}(\theta))=\int_{s-T}^{s} Y^{-1}(\tau, \theta) g(\tau, \widetilde{x}(\tau+\theta)) d \tau, \quad s, \theta \in[0, T] .
$$

We now show that

$$
Y^{-1}(\tau, \theta)=Y(\theta, 0) Y^{-1}(\tau+\theta, 0), \quad \tau, \theta \in[0, T] .
$$

Indeed, it is easy to see that $Y(t+\theta, 0)$ is a fundamental matrix of the system (2.10) and thus $Y(t+\theta, 0) Y^{-1}(\theta, 0)$ is also a fundamental matrix for (2.10); moreover, we have $Y(t+\theta, 0) Y^{-1}(\theta, 0)=I$ for $t=0$. Consequently, $Y(t+\theta, 0) Y^{-1}(\theta, 0)=Y(t, \theta)$, which is equivalent to (2.12). By substituting (2.12) into (2.11) and using the change of variables $\tau+\theta=t$ in the integral of the expression (2.11), we obtain

$$
\begin{aligned}
\widetilde{\Phi}^{s}(\widetilde{x}(\theta)) & =\int_{s-T}^{s} Y^{-1}(\tau, \theta) g(\tau, \widetilde{x}(\tau+\theta)) d \tau \\
& =Y(\theta, 0) \int_{s-T}^{s} Y^{-1}(\tau+\theta, 0) g(\tau, \widetilde{x}(\tau+\theta)) d \tau \\
& =Y(\theta, 0) \int_{s-T+\theta}^{s+\theta} Y^{-1}(t, 0) g(t-\theta, \widetilde{x}(t)) d t .
\end{aligned}
$$

We set $\widetilde{Z}(t)=(\widehat{z}(t), \widetilde{z}(t))$ and let $Z(t)$ denote a fundamental matrix of the conjugate system (2.6) such that $Z(0)=I$; we have $Z(t)=\widetilde{Z}(t) \widetilde{Z}^{-1}(0)$. Henceforth we write $(a, b)$, where $a, b \in \mathbb{R}^{2}$, to denote the matrix whose first column is $a$ and the second is $b$. By Perron's lemma (see [36] or [10, Sec. III, §12]), $Y^{-1}(t)=Z^{*}(t)$ and therefore,

$$
\begin{aligned}
\Phi^{s}(\widetilde{x}(\theta)) & =Y(\theta) \int_{s-T+\theta}^{s+\theta} Y^{-1}(\tau) g(\tau-\theta, \widetilde{x}(\tau)) d \tau \\
& =\left(\widetilde{Z}^{*}(\theta)\right)^{-1} \int_{s-T+\theta}^{s+\theta} \widetilde{Z}^{*}(\tau) g(\tau-\theta, \widetilde{x}(\tau)) d \tau=\left(\widetilde{Z}^{*}(\theta)\right)^{-1}\left(\begin{array}{c}
M_{A}^{s}(\theta) \\
M_{E}^{s}(\theta)
\end{array}\right) .
\end{aligned}
$$

But by observing that

$$
\begin{aligned}
& \left(\widetilde{Z}^{*}(0)\right)^{-1}=\left(\left(\begin{array}{lr}
\left(1 /\|\dot{\widetilde{x}}(0)\|^{2}\right) \dot{\tilde{x}}_{1}(0) & -\dot{\widetilde{x}}_{2}(0) \\
\left(1 /\|\dot{\widetilde{x}}(0)\|^{2}\right) \dot{\widetilde{x}}_{2}(0) & \dot{\widetilde{x}}_{1}(0)
\end{array}\right)^{-1}\right)^{*} \\
& =\frac{1}{\operatorname{det}\left\|\left(\begin{array}{cc}
\left(1 /\|\dot{\widetilde{x}}(0)\|^{2}\right) \dot{\widetilde{x}}_{1}(0) & -\dot{\widetilde{x}}_{2}(0) \\
\left(1 /\|\dot{\widetilde{x}}(0)\|^{2}\right) \dot{\widetilde{x}}_{2}(0) & \dot{\widetilde{x}}_{1}(0)
\end{array}\right)\right\|} \\
& \circ\left(\begin{array}{ll}
\dot{\widetilde{x}}_{1}(0) & \dot{\widetilde{x}}_{2}(0) \\
-\left(1 /\|\dot{\widetilde{x}}(0)\|^{2}\right) \dot{\widetilde{x}}_{2}(0) & \left(1 /\|\dot{\widetilde{x}}(0)\|^{2}\right) \dot{\widetilde{x}}_{1}(0)
\end{array}\right)^{*} \\
& =\left(\begin{array}{lr}
\dot{\widetilde{x}}_{1}(0) & -\left(1 /\|\dot{\widetilde{x}}(0)\|^{2}\right) \dot{\widetilde{x}}_{2}(0) \\
\dot{\widetilde{x}}_{2}(0) & \left(1 /\|\dot{\widetilde{x}}(0)\|^{2}\right) \dot{\widetilde{x}}_{1}(0)
\end{array}\right)=(\dot{\widetilde{x}}(0), \widehat{y}(0)),
\end{aligned}
$$

we conclude that

$$
\left(\widetilde{Z}^{*}(\theta)\right)^{-1}=(\dot{\widetilde{x}}(\theta), \widehat{y}(\theta)) \quad \text { for any } \theta \in \mathbb{R} .
$$

The lemma is proved.

Our next step in proving Theorem 2.1 is to establish that $\operatorname{ind}(\widetilde{x}, \widetilde{\Phi})=\operatorname{ind}(\widetilde{x}, \Phi)$. As in the case of the functions $M_{E}^{s}$ and $M_{A}^{s}$, we assume by definition that

$$
\widetilde{\Phi}:=\widetilde{\Phi}^{0} .
$$


Lemma 2.3. Suppose that condition (A) of Theorem 2.1 holds. Then

$$
\operatorname{ind}(\widetilde{x}, \Phi)=\operatorname{ind}(\widetilde{x}, \widetilde{\Phi})
$$

Proof. We define the deformation

$$
\begin{aligned}
& \Phi_{\lambda}(\widetilde{x}(\theta))=\left(\int_{-T+\lambda \theta}^{\lambda \theta}\langle\widehat{z}(\tau), g(\tau-\theta, \widetilde{x}(\tau))\rangle d \tau\right) \dot{\widetilde{x}}(\theta) \\
& \quad+\left(\int_{-T+\lambda \theta}^{\lambda \theta}\langle\widetilde{z}(\tau), g(\tau-\theta, \widetilde{x}(\tau))\rangle d \tau\right)\left(\lambda \widehat{y}(\theta)+(1-\lambda) \dot{\widetilde{x}}(\theta)^{\perp}\right), \quad \theta \in[0, T] .
\end{aligned}
$$

Since $\Phi_{1}(\widetilde{x}(\theta))=\widetilde{\Phi}(\widetilde{x}(\theta))$ and $\Phi_{0}(\widetilde{x}(\theta))=\Phi(\widetilde{x}(\theta))$, it is sufficient to establish that

$$
\Phi_{\lambda}(\widetilde{x}(\theta)) \neq 0 \quad \text { for all } \lambda \in[0,1], \theta \in[0, T] .
$$

By the definition of $\widehat{y}$ we have $\left\langle\widehat{y}(0), \dot{\widetilde{x}}(0)^{\perp}\right\rangle>0$. But since the vectors $\dot{\widetilde{x}}(\theta)$ and $\widehat{y}(\theta)$ are linearly independent for any $\theta \in[0, T]$, we also have $\left\langle\widehat{y}(\theta), \dot{\widetilde{x}}(\theta)^{\perp}\right\rangle \neq 0$. Hence,

$$
\left\langle\widehat{y}(\theta), \dot{\widetilde{x}}(\theta)^{\perp}\right\rangle>0 \quad \text { for any } \theta \in[0, T]
$$

and therefore,

$$
\left\langle\lambda \widehat{y}(\theta)+(1-\lambda) \dot{\widetilde{x}}(\theta)^{\perp}, \dot{\widetilde{x}}(\theta)^{\perp}\right\rangle>0 \quad \text { for any } \theta \in[0, T], \lambda \in[0, T] .
$$

Suppose that (2.13) is false, that is, that there exist $\lambda_{0} \in[0,1]$ and $\theta_{0} \in[0, T]$ such that

$$
\Phi_{\lambda_{0}}\left(\widetilde{x}\left(\theta_{0}\right)\right)=0
$$

Taking account of (2.14), from the last equality we conclude that

$$
\int_{-T+\lambda_{0} \theta_{0}}^{\lambda_{0} \theta_{0}}\left\langle\widehat{z}(\tau), g\left(\tau-\theta_{0}, \widetilde{x}(\tau)\right)\right\rangle d \tau=0 \quad \text { and } \quad \int_{-T+\lambda_{0} \theta_{0}}^{\lambda_{0} \theta_{0}}\left\langle\widetilde{z}(\tau), g\left(\tau-\theta_{0}, \widetilde{x}(\tau)\right)\right\rangle d \tau=0 .
$$

But $\lambda_{0} \theta_{0} \in[0, T]$ and, consequently, the last relation contradicts the assumption (A).

The lemma is proved.

Finally, we shall need the following result.

Lemma 2.4. A function $x \in C\left([0, T], \mathbb{R}^{2}\right)$ is a T-periodic solution of the system (1.2) if and only if the function

$$
\nu(t)=\Omega(0, t, x(t)), \quad t \in \mathbb{R}
$$

is a solution of the system

$$
\dot{\nu}=\varepsilon \Omega_{(3)}^{\prime}(0, t, \Omega(t, 0, \nu)) g(t, \Omega(t, 0, \nu)), \quad t \in \mathbb{R},
$$

satisfying the condition $\nu(0)=\Omega(T, 0, \nu(T))$.

We note that the system we look at in the statement may have several solutions satisfying the initial condition $\nu(0)=\Omega(T, 0, \nu(T))$. According to the lemma, one of these is always $\nu(t)=\Omega(0, t, x(t))$.

Proof. In the system (1.2) we make the change of variables

$$
x(t)=\Omega(t, 0, \nu(t)) .
$$

Formula (2.16) homeomorphically associates with each function $\nu \in C\left([0, T], \mathbb{R}^{2}\right)$ a function $x \in C\left([0, T], \mathbb{R}^{2}\right)$, and the inverse map is given by formula (2.15). Consequently, a function $x$ is a solution of the system (1.2) if and only if the function $\nu$ defined by (2.15) satisfies the following equality:

$$
\begin{aligned}
& \Omega_{(1)}^{\prime}(t, 0, \nu(t))+\Omega_{(3)}^{\prime}(t, 0, \nu(t)) \dot{\nu}(t) \\
& \quad=\varepsilon g(t, \Omega(t, 0, \nu(t)))+f(\Omega(t, 0, \nu(t))), \quad t \in \mathbb{R} .
\end{aligned}
$$


By the definition of the function $\Omega$ we have

$$
\Omega_{(1)}^{\prime}(t, 0, \nu(t))=f(\Omega(t, 0, \nu(t))) ;
$$

therefore, taking the fact that

$$
\Omega_{(3)}^{\prime}(0, t, \Omega(t, 0, \xi)) \Omega_{(3)}^{\prime}(t, 0, \xi)=I, \quad t \in \mathbb{R}, \xi \in \mathbb{R}^{2},
$$

into account, we can rewrite the system (2.17) in the form

$$
\dot{\nu}(t)=\varepsilon \Omega_{(3)}^{\prime}(0, t, \Omega(t, 0, \nu(t))) g(t, \Omega(t, 0, \nu(t))), \quad t \in \mathbb{R} .
$$

Consider an arbitrary $T$-periodic solution $x$ of the system (1.2). We have

$$
\nu(0)=\Omega(0,0, x(0))=x(0)=x(T)=\Omega(T, 0, \nu(T)) .
$$

The lemma is proved.

We denote the interior of the cycle $\widetilde{x}$ by $U \subset \mathbb{R}^{2}$.

To prove Theorem 2.1 we consider the completely continuous integral operator

$$
Q_{\varepsilon}: C\left([0, T], \mathbb{R}^{2}\right) \rightarrow C\left([0, T], \mathbb{R}^{2}\right)
$$

given by

$$
\left(Q_{\varepsilon} x\right)(t)=x(T)+\int_{0}^{t} f(x(\tau)) d \tau+\varepsilon \int_{0}^{t} g(\tau, x(\tau)) d \tau, \quad t \in[0, T]
$$

on the set

$$
W_{U}=\left\{\widehat{x} \in C\left([0, T], \mathbb{R}^{2}\right): \widehat{x}(t) \in U \text { for any } t \in[0, T]\right\} .
$$

We denote the Leray-Schauder degree of a transformation $I-F: \bar{W} \rightarrow \mathbb{R}^{2}$ with respect to zero by $d(I-F, W)$ (see 20 , Ch. I, $\S 5]$ ). Sometimes, in order to emphasize that $F$ is defined in a space $E$, we shall write $d_{E}(I-F, W)$.

The main role in the proof of Theorem 2.1 is played by the following theorem.

Theorem 2.2. If $\widetilde{\Phi}^{s}(\xi) \neq 0$ for any $\xi \in \partial U$ and any $s \in[0, T]$, then there exists $\varepsilon_{0}>0$ such that for $\varepsilon \in\left(0, \varepsilon_{0}\right]$ the following assertions hold:

1) for any $x \in C\left([0, T], \mathbb{R}^{2}\right)$ such that $Q_{\varepsilon} x=x$, we have $x(t) \notin \partial U$ for all $t \in[0, T]$; in particular, the operator $Q_{\varepsilon}$ has no fixed points on $\partial W_{U}$;

2) $d\left(I-Q_{\varepsilon}, W_{U}\right)=\operatorname{ind}(\widetilde{x}, \widetilde{\Phi})$ for $\varepsilon \in\left(0, \varepsilon_{0}\right]$.

Assertion 2) of Theorem 2.2 was first presented in our Diploma thesis [23] and published in [13] (Theorem 2). The statement of the result on the existence of periodic solutions for the perturbed system (1.2), which follows immediately from assertion 2), was first published in 15 (Theorem 1). Since almost the same work is involved in just proving part 1) as in proving both 1) and 2), it seems appropriate to give the complete proof of Theorem 2.2 below, not the least because this proof is significantly simpler than the one given in 13, where the perturbed system has two summands containing $\varepsilon>0$ and $\varepsilon$ occurs in these summands to different powers.

Theorem 2.2 is a development of the results of Mawhin, who established similar assertions in the case of zero or linear generating systems (see 25] and 26]). Although Mawhin considered the case of the space $\mathbb{R}^{n}$, the proof of Theorem 2.2 extends also immediately to the $n$-dimensional case.

Proof of Theorem 2.2. We set

$$
\Upsilon(t, \xi)=\Omega_{(3)}^{\prime}(0, t, \Omega(t, 0, \xi)) g(t, \Omega(t, 0, \xi)) .
$$


According to Lemma 2.4, to each fixed point of the operator $Q_{\varepsilon}$ in $W_{U}$ there corresponds a fixed point (2.15) of the operator

$$
\left(G_{\varepsilon} \nu\right)(t)=\Omega(T, 0, \nu(T))+\int_{0}^{t} \Upsilon(\tau, \nu(\tau)) d \tau
$$

which, as is easy to verify, again belongs to $W_{U}$. Consequently, if $d\left(I-G_{\varepsilon}, W_{U}\right)$ is defined, then by [16, Theorem 26.4] we have

$$
d\left(I-Q_{\varepsilon}, W_{U}\right)=d\left(I-G_{\varepsilon}, W_{U}\right) .
$$

In the space $C\left([0, T], \mathbb{R}^{2}\right)$ we consider the auxiliary completely continuous operator

$$
\left(A_{\varepsilon} \nu\right)(t)=\Omega(T, 0, \nu(T))-\varepsilon \int_{0}^{T} \Upsilon(\tau, \nu(\tau)) d \tau
$$

and show that for sufficiently small $\varepsilon>0$ the fields $I-G_{\varepsilon}$ and $I-A_{\varepsilon}$ are homotopic on the boundary of the set $W_{U}$. We define the deformation

$$
D_{\varepsilon}(\lambda, \nu)(t)=\nu(t)-\Omega(T, 0, \nu(T))-\varepsilon \int_{0}^{\lambda t+(1-\lambda) T} \Upsilon(\tau, \nu(\tau)) d \tau,
$$

which connects the fields $G_{\varepsilon}$ and $G_{1, \varepsilon}$. We now show that for sufficiently small $\varepsilon>0$ the deformation $D_{\varepsilon}$ is nondegenerate on the boundary of the set $W_{U}$. To do this we prove a stronger assertion, which will then be used in the proof of assertion 1); namely, we shall show that there exists $\varepsilon_{0}>0$ such that for all $\varepsilon \in\left(0, \varepsilon_{0}\right]$ and $\lambda \in[0,1]$ each solution of the equation $D_{\varepsilon}(\lambda, \nu)=\nu$ satisfies the condition $\nu(t) \notin \partial U$ for all $t \in[0, T]$. Suppose not. Then for an arbitrary sequence $\left\{\varepsilon_{k}\right\}_{k=1}^{\infty} \subset(0,1]$ such that $\varepsilon \rightarrow 0$ as $k \rightarrow \infty$, there exist sequences $\left\{\lambda_{k}\right\}_{k=1}^{\infty} \subset[0,1]$ and $\left\{\nu_{k}\right\}_{k=1}^{\infty} \subset C\left([0, T], \mathbb{R}^{2}\right)$ such that

$$
\nu_{k}(t)=\Omega\left(T, 0, \nu_{k}(T)\right)+\varepsilon_{k} \int_{0}^{\lambda_{k} t+\left(1-\lambda_{k}\right) T} \Upsilon\left(\tau, \nu_{k}(\tau)\right) d \tau, \quad t \in[0, T]
$$

and

$$
\nu_{k}([0, T]) \cap \partial U \neq \varnothing .
$$

Since the sequence of numbers $\left\{\lambda_{k}\right\}_{k=1}^{\infty}$ is bounded, we can extract a convergent subsequence. Hence we can assume without loss of generality that the sequence $\left\{\lambda_{k}\right\}_{k=1}^{\infty}$ converges. It follows from (2.21) that the functions of the sequence $\left\{\nu_{k}\right\}_{k=1}^{\infty}$ are uniformly bounded. Therefore, since the function $\Upsilon$ is continuous, there exists a constant $c_{0}>0$ such that $\left\|\Upsilon\left(t, \nu_{k}(t)\right)\right\| \leq c_{0}$ for $t \in[0, T], k \in \mathbb{N}$, and for any $t_{1}, t_{2} \in[0, T], k \in \mathbb{N}$, we have the estimate

$$
\left\|\nu_{k}\left(t_{2}\right)-\nu_{k}\left(t_{1}\right)\right\|=\varepsilon_{k}\left\|\int_{\lambda_{k} t_{1}+\left(1-\lambda_{k}\right) T}^{\lambda_{k} t_{2}+\left(1-\lambda_{k} T\right)} \Upsilon\left(\tau, z_{k}(\tau)\right) d \tau\right\| \leq \varepsilon_{k} \lambda_{k}\left(t_{2}-t_{1}\right) c_{0},
$$

which implies that the functions of the sequence $\left\{\nu_{k}\right\}_{k=1}^{\infty}$ are equicontinuous. Therefore, applying Arzelà's theorem we can extract a convergent subsequence from this sequence. Hence we can assume without loss of generality that the sequence $\left\{\nu_{k}\right\}_{k=1}^{\infty}$ converges. We set $\lambda_{0}=\lim _{k \rightarrow \infty} \lambda_{k}$ and $\nu_{0}=\lim _{k \rightarrow \infty} \nu_{k}$. Then $\lambda_{0} \in[0,1]$ and $\nu_{0}([0, T]) \cap \partial U \neq \varnothing$. Since $\dot{\nu}_{k} \rightarrow 0$ as $k \rightarrow \infty$, the function $\nu_{0}$ is constant. Relation (2.21) is equivalent to the existence of a number $t_{k} \in[0, T]$ such that $\nu_{k}\left(t_{k}\right) \in \partial U$. Then

$$
\Omega\left(T, 0, \nu_{k}\left(t_{k}\right)\right)=\nu_{k}\left(t_{k}\right), \quad k \in \mathbb{N} .
$$

Subtracting equality (2.20) written for $t=t_{k}$ from the same equality written for $t=T$, we obtain

$$
\nu_{k}(T)-\nu_{k}\left(t_{k}\right)=\varepsilon_{k} \int_{\lambda_{k} t_{k}+\left(1-\lambda_{k}\right) T}^{T} \Upsilon\left(\tau, \nu_{k}(\tau)\right) d \tau
$$


Based on (2.22), the expression (2.20) for $t=T$ can be rewritten in the form

or

$$
\nu_{k}(T)-\nu_{k}\left(t_{k}\right)=\Omega\left(T, 0, \nu_{k}(T)\right)-\Omega\left(T, 0, \nu_{k}\left(t_{k}\right)\right)+\varepsilon_{k} \int_{0}^{T} \Upsilon\left(\tau, z_{k}(\tau)\right) d \tau
$$

$$
\begin{aligned}
(I- & \left.\Omega_{(3)}^{\prime}\left(T, 0, \nu_{k}\left(t_{k}\right)\right)\right)\left(\nu_{k}(T)-\nu_{k}\left(t_{k}\right)\right) \\
& =\varepsilon_{k} \int_{0}^{T} \Upsilon\left(\tau, \nu_{k}(\tau)\right) d \tau+o\left(\nu_{k}\left(t_{k}\right), \nu_{k}(T)-\nu_{k}\left(t_{k}\right)\right),
\end{aligned}
$$

where the function $o(\xi, h)$ satisfies the relation

$$
\frac{\|o(\xi, h)\|}{\|h\|} \rightarrow 0 \quad \text { as } \mid h \| \rightarrow 0 \quad \text { for } \xi \in \mathbb{R}^{2} .
$$

Substituting (2.23) into (2.24) we obtain the equality

$$
\begin{aligned}
(I- & \left.\Omega_{(3)}^{\prime}\left(T, 0, \nu_{k}\left(t_{k}\right)\right)\right) \int_{\lambda_{k} t_{k}+\left(1-\lambda_{k}\right) T}^{T} \Upsilon\left(\tau, \nu_{k}(\tau)\right) d \tau \\
& =\int_{0}^{T} \Upsilon\left(\tau, \nu_{k}(\tau)\right) d \tau+\frac{o\left(\nu_{k}\left(t_{k}\right), \nu_{k}(T)-\nu_{k}\left(t_{k}\right)\right)}{\varepsilon_{k}} .
\end{aligned}
$$

It follows from (2.23) that there exists a constant $c>0$ such that

$$
\left\|\nu_{k}(T)-\nu_{k}\left(t_{k}\right)\right\| \leq c \varepsilon_{k} .
$$

Hence,

$$
\left\|\frac{o\left(\nu_{k}\left(t_{k}\right), \nu_{k}(T)-\nu_{k}\left(t_{k}\right)\right)}{\varepsilon_{k}}\right\| \leq c \frac{\left\|o\left(\nu_{k}\left(t_{k}\right), \nu_{k}(T)-\nu_{k}\left(t_{k}\right)\right)\right\|}{\left\|\nu_{k}(T)-\nu_{k}\left(t_{k}\right)\right\|} .
$$

In view of the fact that the values of the functions $\nu_{k}$ are bounded uniformly in $k \in \mathbb{N}$, it follows from (2.27) that

$$
\left\|\frac{o\left(\nu_{k}\left(t_{k}\right), \nu_{k}(T)-\nu_{k}\left(t_{k}\right)\right)}{\varepsilon_{k}}\right\| \rightarrow 0 \quad \text { as } k \rightarrow \infty .
$$

Taking (2.28) into account we pass to the limit in (2.26) as $k \rightarrow \infty$ and obtain

or

$$
\left(I-\Omega_{(3)}^{\prime}\left(T, 0, \xi_{0}\right)\right) \int_{s}^{T} \Upsilon\left(\tau, \xi_{0}\right) d \tau=\int_{0}^{T} \Upsilon\left(\tau, \xi_{0}\right) d \tau
$$

$$
\begin{gathered}
\int_{s}^{T} \Omega_{(3)}^{\prime}\left(T, 0, \xi_{0}\right) \Omega_{(3)}^{\prime}\left(0, \tau, \Omega\left(\tau, 0, \xi_{0}\right)\right) g\left(\tau, \Omega\left(\tau, 0, \xi_{0}\right)\right) d \tau \\
-\int_{s}^{0} \Omega_{(3)}^{\prime}\left(0, \tau, \Omega\left(\tau, 0, \xi_{0}\right)\right) g\left(\tau, \Omega\left(\tau, 0, \xi_{0}\right)\right) d \tau=0,
\end{gathered}
$$

where $s=\lim _{k \rightarrow \infty}\left(\lambda_{k} t_{k}+\left(1-\lambda_{k}\right) T\right) \in[0, T]$. Using Lemma 2.2] we can rewrite (2.29) in the form

$$
\eta\left(T, s, \xi_{0}\right)-\eta\left(0, s, \xi_{0}\right)=0
$$

which contradicts the hypothesis of the theorem. Thus, there exists $\varepsilon_{0}>0$ such that for all $\varepsilon \in\left(0, \varepsilon_{0}\right]$ and $\lambda \in[0,1]$, each solution of the equation $D_{\varepsilon}(\lambda, \nu)=\nu$ satisfies the condition $\nu(t) \notin \partial U$ for all $t \in[0, T]$. For $\lambda=1$, the result obtained coincides with assertion 1) of the theorem. We now turn to the proof of assertion 2). As we already mentioned, the property we have proved means, in particular, that

$$
D_{\varepsilon}(\lambda, \nu) \neq 0, \quad \lambda \in[0,1], \nu \in \partial W_{U}, \varepsilon \in\left(0, \varepsilon_{0}\right],
$$


that is, that the fields $I-G_{\varepsilon}$ and $I-A_{\varepsilon}$ are homotopic on the boundary of the set $W_{U}$ for $\varepsilon \in\left(0, \varepsilon_{0}\right]$. We denote by $C_{0}\left([0, T], \mathbb{R}^{2}\right)$ the subspace of the space $C\left([0, T], \mathbb{R}^{2}\right)$ consisting of all the constant functions defined on the segment $[0, T]$ and taking values in $\mathbb{R}^{2}$. We have $A_{\varepsilon}\left(\partial W_{U}\right) \subset C_{0}\left([0, T], \mathbb{R}^{2}\right)$. Next, by construction, the set $W_{U}$ contains functions identically equal to an arbitrary fixed element of $U$. Finally, from (2.30) for $\lambda=0$ we obtain

$$
A_{\varepsilon}(\nu) \neq \nu, \quad \nu \in \partial W_{U}, \varepsilon \in\left(0, \varepsilon_{0}\right],
$$

whence, in view of the relation $\partial\left(W_{U} \cap C_{0}\left([0, T], \mathbb{R}^{2}\right)\right) \subset \partial W_{U}$, it follows that for $\varepsilon \in\left(0, \varepsilon_{0}\right]$ the field $I-A_{\varepsilon}$ has no zeros on the boundary of the set $W_{U} \cap C_{0}\left([0, T], \mathbb{R}^{2}\right)$. Therefore for $\varepsilon \in\left(0, \varepsilon_{0}\right]$ it is legitimate to restrict the field $I-A_{\varepsilon}$ to the subspace $C_{0}\left([0, T], \mathbb{R}^{2}\right)$, which means that

$$
d_{C\left([0, T], \mathbb{R}^{2}\right)}\left(I-A_{\varepsilon}, W_{U}\right)=d_{C_{0}\left([0, T], \mathbb{R}^{2}\right)}\left(I-A_{\varepsilon}, W_{U} \cap C_{0}\left([0, T], \mathbb{R}^{2}\right)\right),
$$

where on the left- and right-hand sides of the equality there are the topological degrees in the spaces $C\left([0, T], \mathbb{R}^{2}\right)$ and $C_{0}\left([0, T], \mathbb{R}^{2}\right)$, respectively.

We observe that a constant function $\nu \in W_{U} \cap C_{0}\left([0, T], \mathbb{R}^{2}\right)$ is a solution of the equation $A_{\varepsilon} \nu=\nu$ if and only if the element $\xi=\nu(0)$ is a solution of the equation $A_{\varepsilon}^{0} \xi=\xi$, where

$$
A_{\varepsilon}^{0} \xi=\Omega(T, 0, \xi)+\varepsilon \int_{0}^{T} \Upsilon(\tau, \xi) d \tau .
$$

Applying the theorem on equivalent equations to the equations $A_{\varepsilon} \nu=\nu$ and $A_{\varepsilon}^{0} \xi=\xi$, and then applying the theorem on passing to a subspace, for $\varepsilon \in\left(0, \varepsilon_{0}\right]$ we obtain

$$
d_{C_{0}\left([0, T], \mathbb{R}^{2}\right)}\left(I-A_{\varepsilon}, W_{U} \cap C_{0}\left([0, T], \mathbb{R}^{2}\right)\right)=d_{\mathbb{R}^{2}}\left(I-A_{\varepsilon}^{0}, U\right) .
$$

In order to calculate the topological degree $d_{\mathbb{R}^{2}}\left(I-A_{\varepsilon}^{0}, U\right)$ we set

$$
A_{1, \varepsilon} \xi=-\varepsilon \int_{0}^{T} \Upsilon(\tau, \xi) d \tau
$$

Since $\left(I-A_{\varepsilon}^{0}\right)(\xi)=A_{1, \varepsilon} \xi, \quad \xi \in \partial U$, for $\varepsilon \in\left(0, \varepsilon_{0}\right]$ we have

$$
d_{\mathbb{R}^{2}}\left(I-A_{\varepsilon}^{0}, U\right)=d_{\mathbb{R}^{2}}\left(A_{1, \varepsilon}, U\right) .
$$

We claim that the vector fields $A_{1, \varepsilon}$ and $A_{1,1}$ are homotopic on the boundary of the set $U$ for $\varepsilon \in\left(0, \varepsilon_{0}\right]$. We define the linear deformation

$$
D_{1, \varepsilon}(\lambda, \xi)=(\lambda \varepsilon+1-\lambda) \int_{0}^{T} \Upsilon(\tau, \xi) d \tau, \quad \xi \in U
$$

and establish that it is nondegenerate on the boundary of the set $U$. Suppose the opposite; then for some $\lambda \in[0,1], \quad \xi \in \partial U$, and $\varepsilon \in\left(0, \varepsilon_{0}\right)$ we have

$$
\left(\lambda_{0} \varepsilon+1-\lambda\right) \int_{0}^{T} \Upsilon(\tau, \xi) d \tau=0,
$$

whence

$$
\int_{0}^{T} \Upsilon(\tau, \xi) d \tau=0
$$

which, by Lemma 2.2, contradicts the hypotheses of the theorem. Thus, using Lemma 2.2 we have

$$
d_{\mathbb{R}^{2}}\left(A_{1, \varepsilon}, U\right)=d_{\mathbb{R}^{2}}\left(A_{1,1}, U\right)=d_{\mathbb{R}^{2}}(-\eta(T, 0, \cdot), U) .
$$

Substituting (2.34) into (2.33) we obtain

$$
d_{\mathbb{R}^{2}}\left(I-A_{\varepsilon}^{0}, U\right)=d_{\mathbb{R}^{2}}(-\eta(T, 0, \cdot), U), \quad \varepsilon \in\left(0, \varepsilon_{0}\right) .
$$


Now, substituting (2.32) into (2.31), using the fact that the fields $I-A_{\varepsilon}$ and $I-G_{\varepsilon}$ are homotopic, and using relation (2.35), we finally have

$$
d_{C\left([0, T], \mathbb{R}^{2}\right)}\left(I-G_{\varepsilon}, W_{U}\right)=d_{\mathbb{R}^{2}}(-\eta(T, 0, \cdot), U), \quad \varepsilon \in\left(0, \varepsilon_{0}\right] .
$$

But the field $\eta(T, 0, \cdot)$ is obtained from the field $-\eta(T, 0, \cdot)$ by continuous rotation of all the vectors through $180^{\circ}$ counterclockwise; consequently,

$$
d_{\mathbb{R}^{2}}(-\eta(T, 0, \cdot), U)=d_{\mathbb{R}^{2}}(\eta(T, 0, \cdot), U) .
$$

Since the topological degree is independent of the way it is defined (see [16]), by the definition of the set $U$ we have $d_{\mathbb{R}^{2}}(\eta(T, 0, \cdot), U)=\operatorname{ind}(\widetilde{x}, \eta(T, 0, \cdot))=\operatorname{ind}(\widetilde{x}, \widetilde{\Phi})$.

The theorem is proved.

Everything is now in place for us to prove Theorem 2.1

Proof of Theorem 2.1. Let $\varepsilon_{0}>0$ be as in Theorem 2.2. then, taking Lemmas 2.1 and 2.3 into account as well, we have

$$
d\left(Q_{\varepsilon}, W_{U}\right)=\operatorname{ind}(\widetilde{x}, \Phi) \quad \text { for any } \varepsilon \in\left(0, \varepsilon_{0}\right]
$$

and, using assumption $(\underline{B})$, we obtain

$$
d\left(Q_{\varepsilon}, W_{U}\right) \neq 1 \quad \text { for any } \varepsilon \in\left(0, \varepsilon_{0}\right] .
$$

We set $U_{\delta}^{-}=U \backslash B_{\delta}(\partial U)$ and $U_{\delta}^{+}=U \cup B_{\delta}(\partial U)$. Henceforth, $B_{\delta}(D)$ denotes the $\delta$-neighbourhood of a set $D$ in the norm of the space containing $D$. Based on condition $(C)$ we can fix $\delta_{0}>0$ such that the generating system (1.1) has no $T$-periodic solutions with initial conditions in $\partial U_{\delta}^{-} \cup \partial U_{\delta}^{+}$for all $\delta \in\left(0, \delta_{0}\right]$. We can assume without loss of generality that $\delta_{0}>0$ is chosen to be sufficiently small, so that $U_{\delta}^{-} \neq \varnothing$. By the Capietto-Mawhin-Zanolin theorem ([5, Corollary 1]) we have

$$
d\left(Q_{0}, W_{U_{\delta}^{-}}\right)=d_{\mathbb{R}^{2}}\left(f, U_{\delta}^{-}\right) \quad \text { and } \quad d\left(Q_{0}, W_{U_{\delta}^{+}}\right)=d_{\mathbb{R}^{2}}\left(f, U_{\delta}^{+}\right) \quad \text { for all } \delta \in\left(0, \delta_{0}\right] .
$$

We can assume without loss of generality that $\delta_{0}>0$ is small enough for the following equalities to hold:

$$
d_{\mathbb{R}^{2}}\left(f, U_{\delta}^{-}\right)=d_{\mathbb{R}^{2}}\left(f, U_{\delta}^{+}\right)=d_{\mathbb{R}^{2}}(f, U), \quad \delta \in\left(0, \delta_{0}\right] .
$$

By Poincaré's theorem (see Lefschetz [21, Theorem 11.1] or Krasnosel'skil et al. [18, Theorem 2.3]) we have $d_{\mathbb{R}^{2}}(f, U)=1$; therefore,

$$
d\left(Q_{0}, W_{U_{\delta}^{-}}\right)=d\left(Q_{0}, W_{U_{\delta}^{+}}\right)=1 \quad \text { for all } \delta \in\left(0, \delta_{0}\right] .
$$

Thus, to each $\delta \in\left(0, \delta_{0}\right]$ there corresponds $\varepsilon_{\delta}>0$ such that

$$
d\left(Q_{\varepsilon}, W_{U_{\delta}^{-}}\right)=d\left(Q_{\varepsilon}, W_{U_{\delta}^{+}}\right)=1 \quad \text { for all } \varepsilon \in\left(0, \varepsilon_{\delta}\right] \text { and } \delta \in\left(0, \delta_{0}\right] .
$$

We can assume without loss of generality that $\varepsilon_{\delta}<\varepsilon_{0}$ for all $\delta \in\left(0, \delta_{0}\right]$. Then from (2.36) and (2.37) we obtain that for all $\delta \in\left(0, \delta_{0}\right]$ and $\varepsilon \in\left(0, \varepsilon_{\delta}\right]$ the system (1.2) has at least two $T$-periodic solutions $x_{\varepsilon, 1} \in W_{U} \backslash W_{U_{\delta}^{-}}$and $x_{\varepsilon, 2} \in W_{U_{\delta}^{+}} \backslash W_{U}$. Hence, in particular, we have $x_{\varepsilon, 1}(0) \in U$ and $x_{\varepsilon, 2}(0) \notin U$; using assertion 1) of Theorem 2.2, we can conclude that $x_{\varepsilon, 1}(t) \in U$ and $x_{\varepsilon, 2}(t) \notin U$ for all $t \in[0, T]$.

The theorem is proved. 


\section{Calculating the index}

In this section we show that if the function $M$ has exactly two zeros on the interval $[0, T)$, then verifying condition $(B)$ in Theorem 2.1 , that the Poincaré index is distinct from +1 , reduces to verifying an algebraic inequality. The following result will be a basic tool.

Theorem 3.1. Suppose that the perturbation in (1.2) is continuous. Suppose that the function $M_{E}$ has exactly two zeros $\theta_{1}$ and $\theta_{2}$ on the interval $[0, T)$. If $M_{E}$ is strictly monotonic at the points $\theta_{1}$ and $\theta_{2}$ and

$$
M_{A}\left(\theta_{1}\right) \cdot M_{A}\left(\theta_{2}\right)<0
$$

then either $\operatorname{ind}(\widetilde{x}, \Phi)=0$ or $\operatorname{ind}(\widetilde{x}, \Phi)=2$.

The proof of the theorem is based on the following lemma.

Lemma 3.1 (see [22, Lemma 5]). Let $q:[0, T] \rightarrow \mathbb{R}^{2}, q(0)=q(T)$, be a Jordan curve, and $\psi: \mathbb{R}^{2} \rightarrow \mathbb{R}^{2}$ a continuous vector field such that $\psi(q(t)) \neq 0$ for each $t \in[0, T]$. Suppose that there exists a direction function $z:[0, T] \rightarrow \mathbb{R}^{2}, z(0)=z(T)$, such that

1) $\langle z(\theta), \dot{q}(\theta)\rangle \neq 0$ for each $\theta \in[0, T]$;

2) the scalar function $\alpha(\theta)=\langle\psi(q(\theta)), z(\theta)\rangle$ has exactly two zeros $\theta_{1}, \theta_{2}$ on the interval $[0, T)$ and is strictly monotonic at these points;

$3) \operatorname{sign}\left\langle\psi\left(q\left(\theta_{1}\right)\right),\left(\begin{array}{r}z_{2}\left(\theta_{1}\right) \\ -z_{1}\left(\theta_{1}\right)\end{array}\right)\right\rangle=-\operatorname{sign}\left\langle\psi\left(q\left(\theta_{2}\right)\right),\left(\begin{array}{r}z_{2}\left(\theta_{2}\right) \\ -z_{1}\left(\theta_{2}\right)\end{array}\right)\right\rangle$.

Then either $\operatorname{ind}(q, \psi)=0$ or $\operatorname{ind}(q, \psi)=2$.

We point out that Lemma 3.1 is a refinement of the Borsuk-Ulam theorem on even vector fields [3] for the special case under consideration.

Proof of Theorem 3.1. We use Lemma 3.1. For that we choose $q=\widetilde{x}, \psi=\Phi, z=\dot{\widetilde{x}}$. We have

$$
\alpha(\theta)=-M_{E}(\theta)
$$

and

$$
\left\langle\Phi(\widetilde{x}(\theta)),\left(\begin{array}{c}
\dot{\widetilde{x}}_{2}(\theta) \\
-\dot{\widetilde{x}}_{1}(\theta)
\end{array}\right)\right\rangle=-\left\langle\Phi(\widetilde{x}(\theta)), \dot{\widetilde{x}}(\theta)^{\perp}\right\rangle=-M_{A}(\theta) .
$$

In other words, the hypotheses of Lemma 3.1 coincide with the assumptions of this theorem, which completes the proof.

Remark 3.1. If, in the statement of Theorem 3.1, $M_{A}$ is replaced by $M_{E}$ and $M_{E}$ by $M_{A}$, then the resulting theorem holds.

Remark 3.2. Results similar to Theorem 3.1 can be proved in the case where the function $M_{E}$ has an arbitrary number of zeros. In this case, we have to assume that the sign of the function $M_{A}$ alternates appropriately at these zeros.

\section{A comparison with the theorems of Malkin and Mel'nikov}

First of all we establish that the function $M_{A}$ coincides with Malkin's function in the case where Malkin's conditions $\left(C_{M A}\right)$ hold, and the function $M_{E}$ with Mel'nikov's function in the case where Mel'nikov's conditions $\left(C_{M E}\right)$ and some additional symmetry properties hold. 
In [24], under the assumption of $\left(C_{M A}\right)$, Malkin defined the bifurcation function $\widetilde{M}_{A}$ as (see [24, formula 3.13])

$$
\widetilde{M}_{A}(\theta)=\int_{0}^{T}\langle\widetilde{\widetilde{z}}(\tau), g(\tau-\theta, \widetilde{x}(\tau))\rangle d \tau,
$$

where $\widetilde{z}$ is a $T$-periodic solution of the conjugate system (2.6) satisfying the condition

$$
\langle\widetilde{\widetilde{z}}(0), \dot{\widetilde{x}}(0)\rangle=1 \text {. }
$$

In 24] they point out that this choice is always possible (see [24, formulae 2.13 and 3.7]). Under condition $\left(C_{M A}\right)$ this choice is necessarily unique; that is, Malkin's function is defined uniquely. In the same paper, Malkin presents the following result (see [24]).

Malkin's Theorem. Suppose that the right-hand side of the perturbed system (1.2) is continuously differentiable and that Malkin's condition $\left(C_{M A}\right)$ holds. If for sufficiently small $\varepsilon>0$ the perturbed system (1.2) has a T-periodic solution $\widetilde{x}_{\varepsilon}$ such that

$$
\widetilde{x}_{\varepsilon}(t) \rightarrow \widetilde{x}^{\theta_{0}}(t) \quad \text { as } \varepsilon \rightarrow 0,
$$

then

$$
\widetilde{M}_{A}\left(\theta_{0}\right)=0 .
$$

If, along with the indicated necessary condition, the zero $\theta_{0}$ is simple, that is, $\widetilde{M}_{A}{ }^{\prime}\left(\theta_{0}\right)$ $\neq 0$, then for all sufficiently small $\varepsilon>0$ the perturbed system (1.2) does have a T-periodic solution $\widetilde{x}_{\varepsilon}$ satisfying (4.39).

The following assertion gives conditions under which $M_{A}^{s}(\theta)$ is independent of $s$ and coincides with $\widetilde{M}_{A}$.

Lemma 4.1. Suppose that Malkin's condition $\left(C_{M A}\right)$ holds. If $\widehat{y}$ is an eigenfunction of the linearized system (1.4), then the solution $\widehat{z}$ is T-periodic. In particular,

$$
M_{A}^{s}(\theta)=\widetilde{M}_{A}(\theta) \quad \text { for all } s, \theta \in[0, T] .
$$

Proof. Let $\widetilde{\widetilde{z}}$ be a $T$-periodic function involved in the definition of the function $\widetilde{M}_{A}$ and, consequently, satisfying (4.38). Since $\widehat{y}$ is an eigenfunction of the system (1.4), in view of condition $\left(C_{M A}\right)$ there exists $\rho \neq 1$ such that

$$
\widehat{y}(T)=\rho \widehat{y}(0) .
$$

But by Perron's lemma (see [36] or [10, Sec. III, $\S 12]$ ),

$$
\langle\widetilde{\widetilde{z}}(0), \widehat{y}(0)\rangle=\langle\widetilde{\widetilde{z}}(T), \widehat{y}(T)\rangle,
$$

which is possible only in the case where

$$
\langle\widetilde{\widetilde{z}}(0), \widehat{y}(0)\rangle=0
$$

On the other hand, by the definition of $\widehat{z}$ we have

$$
\langle\widehat{z}(0), \dot{\widetilde{x}}(0)\rangle=1 \quad \text { and } \quad\langle\widehat{z}(0), \widehat{y}(0)\rangle=0 .
$$

Thus, the inner products of the vectors $\widehat{z}(0)$ and $\widetilde{\widetilde{z}}(0)$ both with the vector $\dot{\widetilde{x}}(0)$ and with the vector $\widehat{y}(0)$ take the same values. Consequently, $\widehat{z}(0)=\widetilde{z}(0)$.

The lemma is proved.

Thus, if the hypotheses of Lemma 4.1 hold, then it is easier to verify the hypotheses of Theorem 2.1.

We now turn to Mel'nikov's case $\left(C_{M E}\right)$. In [27, Mel'nikov introduced the function

$$
\widetilde{M}_{E}(\theta)=\int_{0}^{T} \operatorname{det}\|(\dot{\widetilde{x}}(\tau), g(\tau-\theta, \widetilde{x}(\tau)))\| d \tau
$$


and established the following result (see [27, Lemma 7]).

Mel'nikov's Theorem. Suppose that the right-hand side of the perturbed system (1.2) is twice continuously differentiable and that the cycle $\widetilde{x}$ is embedded into some family of cycles of the generating Hamiltonian system (1.1). Suppose that Mel'nikov's condition $\left(C_{M E}\right)$ holds. If for sufficiently small $\varepsilon>0$ the perturbed system (1.2) has a T-periodic solution $\widetilde{x}_{\varepsilon}$ converging to the cycle $\widetilde{x}$ as $\varepsilon \rightarrow 0$ up to a shift, then there exists $\theta_{0} \in[0, T]$ such that

$$
\widetilde{M}_{E}\left(\theta_{0}\right)=0 .
$$

If, along with the necessary condition indicated, the zero $\theta_{0}$ is simple, that is, $\widetilde{M}_{E}^{\prime}\left(\theta_{0}\right) \neq 0$, then for all sufficiently small $\varepsilon>0$ the perturbed system (1.2) does have a T-periodic solution $\widetilde{x}_{\varepsilon}$ such that (4.39) holds.

In fact, in the paper cited above, Mel'nikov considered the case of analytic right-hand sides, but for the assertion that we are interested in, it is indeed sufficient to require that they be twice continuously differentiable; the corresponding statement and proof can be found, for example, in [9, Theorem 4.6.2].

It turns out that the conditions for the function $M_{E}^{s}$ to be independent of $s$ and to coincide with $\widetilde{M}_{E}$ are not the same. We will now derive these conditions.

First we establish one auxiliary result.

Lemma 4.2. Suppose that a T-periodic system

$$
\dot{u}=A(t) u, \quad u \in \mathbb{R}^{2}
$$

has multiplicator +1 of algebraic multiplicity 2 , and let $\widetilde{u}$ be a $T$-periodic solution of this system such that

$$
\widetilde{u}_{1}(0)=0, \quad \widetilde{u}_{2}(0) \neq 0 .
$$

Then for a solution $\widehat{u}$ of the system (4.40) satisfying the condition

$$
\widehat{u}_{1}(0) \neq 0, \quad \widehat{u}_{2}(0)=0,
$$

we have the formula

$$
\widehat{u}(t+T)=\widehat{u}(t)+\frac{\widehat{u}_{2}(T)}{\widetilde{u}_{2}(0)} \widetilde{u}(t), \quad t \in \mathbb{R} .
$$

Proof. We denote by $X$ a normalized $(X(0)=I)$ fundamental matrix of the system (4.40). Since

$$
X\left(T_{0}\right)\left(\begin{array}{l}
0 \\
1
\end{array}\right)=\left(\begin{array}{l}
0 \\
1
\end{array}\right)
$$

we have

$$
X\left(T_{0}\right)=\left(\begin{array}{ll}
a & 0 \\
b & 1
\end{array}\right) .
$$

According to the hypothesis of the lemma, $X(T)$ has the repeated eigenvalue +1 ; therefore, $X\left(T_{0}\right)=\left(\begin{array}{ll}10 & b 1\end{array}\right)$, where $b \in \mathbb{R}$ is some number. We have

$$
\begin{aligned}
X\left(t+T_{0}\right) \widehat{u}(0) & =X(t) X\left(T_{0}\right) \widehat{u}(0)=X(t)\left(\begin{array}{ll}
1 & 0 \\
b & 1
\end{array}\right) \widehat{u}(0) \\
& =X(t) \widehat{u}(0)+X(t)\left(\begin{array}{c}
0 \\
b \widehat{u}_{1}(0)
\end{array}\right)=X(t) \widehat{u}(0)+\frac{b \widehat{u}_{1}(0)}{\widetilde{u}_{2}(0)} \widetilde{u}(t) .
\end{aligned}
$$

At the same time,

$$
X\left(T_{0}\right) \widehat{u}(0)=\left(\begin{array}{ll}
1 & 0 \\
b & 1
\end{array}\right) \widehat{u}(0)=\widehat{u}(0)+\left(\begin{array}{c}
0 \\
b \widehat{u}_{1}(0)
\end{array}\right)
$$


whence $b \widehat{u}_{1}(0)=\widehat{u}_{2}(T)$. The lemma is proved.

The following lemma asserts that Mel'nikov's condition $\left(C_{M E}\right)$ is sufficient for $M_{E}^{s}(\theta)$ to be independent of $s$ (in Malkin's case, additional conditions were needed; see Lemma 4.2).

Lemma 4.3. If the algebraic multiplicity of the multiplier +1 of the linearized system (1.4) is equal to 2 , then the solution $\widetilde{z}$ is T-periodic. In particular, $M_{E}^{s}(\theta)$ is independent of $s$.

The hypothesis of Lemma 4.3 holds both in Mel'nikov's case $\left(C_{M E}\right)$ and in the degenerate case where each solution of the system (1.4) is $T$-periodic.

Proof of Lemma 4.3 . Let $\theta \in[0, T]$ be such that

$$
\dot{\tilde{x}}_{1}(\theta)=0 \text {. }
$$

Then the function $\dot{\vec{x}}^{\theta}$ is a solution of the system

$$
\dot{y}=f^{\prime}(\widetilde{x}(t+\theta)) y
$$

with the initial condition $\dot{\vec{x}}_{1}^{\theta}(0)=0$. We denote by $\widehat{\widehat{y}}$ a solution of the system (4.42) with the initial condition $\widehat{\widehat{y}}(0)=(1,0)$. Applying Lemma 4.2 we conclude that

$$
\widehat{\widehat{y}}(T)=\widehat{\widehat{y}}(0)+\frac{\widehat{\widehat{y}}_{2}(T)}{\dot{\widetilde{x}}_{2} \theta(0)} \dot{\widetilde{x}}^{\theta}(T) .
$$

If $\widehat{\hat{y}}_{2}(T)=0$, then from (4.43) each solution of the system (4.42), and therefore also of the system (2.6), is $T$-periodic. We consider the case where

$$
\widehat{\widehat{y}}_{2}(T) \neq 0 \text {. }
$$

By the theorem on periodic solutions of the conjugate system (see [10, Ch. III, $\S 23$, Theorem 2]), the system

$$
\dot{z}=-\left(f^{\prime}(\widetilde{x}(t+\theta))\right)^{*} z
$$

has at least one $T$-periodic solution; we denote this solution by $\widetilde{\widetilde{z}}$. Taking the inner product of (4.43) with $\widetilde{\widetilde{z}}$ we obtain

$$
\begin{aligned}
\left\langle\widehat{y}^{\theta}(T), \widetilde{\widetilde{z}}(T)\right\rangle & =\left\langle\widehat{y}^{\theta}(0), \widetilde{\widetilde{z}}(T)\right\rangle+\frac{\widehat{y}_{2}^{\theta} a(T)}{\dot{\tilde{x}}_{2}^{\theta}(0)}\left\langle\dot{\tilde{x}}^{\theta}(t), \widetilde{\widetilde{z}}(T)\right\rangle \\
& =\left\langle\widehat{y}^{\theta}(0), \widetilde{\widetilde{z}}(0)\right\rangle+\frac{\widehat{y}_{2}^{\theta}(T)}{\dot{\tilde{x}}_{2}^{\theta}(0)} \dot{\tilde{x}}_{2}^{\theta}(0) \widetilde{\widetilde{z}}_{2}(0) .
\end{aligned}
$$

But by Perron's lemma (see [36] or [10, Sec. III, §12]),

$$
\left\langle\widehat{y}^{\theta}(T), \widetilde{\widetilde{z}}(T)\right\rangle=\left\langle\widehat{y}^{\theta}(0), \widetilde{\widetilde{z}}(0)\right\rangle ;
$$

therefore $\widetilde{\widetilde{z}}_{2}(0)=0$. Taking (4.41) into account we conclude that $\left\langle\dot{\tilde{x}}^{\theta}(0), \widetilde{\widetilde{z}}(0)\right\rangle=0$ or

$$
\left\langle\dot{\widetilde{x}}(0), \widetilde{\widetilde{z}}^{-\theta}(0)\right\rangle=0 \text {. }
$$

Consequently, the vectors $\widetilde{\widetilde{z}}^{-\theta}(0)$ and $\widetilde{z}(0)$ are linearly dependent; that is, there exists $a \neq 0$, such that $\widetilde{z}(0)=a \widetilde{\widetilde{z}}^{-\theta}(0)$. But both the functions $\widetilde{\widetilde{z}}^{-\theta}$ and $\widetilde{z}$ are solutions of exactly the same conjugate system (2.6); therefore,

$$
\widetilde{z}(t)=a \widetilde{\widetilde{z}}^{-\theta}(t) \quad \text { for all } t \in[0, T]
$$

in particular, the function $\widetilde{z}$ is $T$-periodic, and the lemma is proved. 
Finally, we produce conditions under which $M_{E}=\widetilde{M}_{E}$.

Lemma 4.4. Suppose that Mel'nikov's condition $\left(C_{M E}\right)$ holds. Suppose that for all $\xi \in \mathbb{R}^{2}$ we have

$$
\begin{aligned}
f_{1}(\xi) & =f_{1}\left(-\xi_{1}, \xi_{2}\right), \\
f_{2}(\xi) & =-f_{2}\left(-\xi_{1}, \xi_{2}\right), \\
\left(f_{1}\right)_{(1)}^{\prime}(\xi) & =-\left(f_{2}\right)_{(2)}^{\prime}(\xi) .
\end{aligned}
$$

Then for any $\theta \in \mathbb{R}$ we have

$$
\widehat{z}(\theta)=\left(\begin{array}{r}
\widehat{y}_{2}(\theta) \\
-\widehat{y}_{1}(\theta)
\end{array}\right), \quad \widetilde{z}(\theta)=\left(\begin{array}{r}
-\dot{\widetilde{x}}_{2}(\theta) \\
\dot{\widetilde{x}}_{1}(\theta)
\end{array}\right)
$$

and, in particular,

$$
\begin{aligned}
& M_{E}^{s}(\theta)=\widetilde{M}_{E}(\theta), \\
& M_{A}^{s}(\theta)=-\int_{s-T}^{s} \operatorname{det}\|(\widehat{y}(\tau), g(\tau-\theta, \widetilde{x}(\tau)))\| d \tau, \quad s, \theta \in[0, T] .
\end{aligned}
$$

To prove Lemma 4.4 we use the following result.

Lemma 4.5. Consider the linear system

$$
\left(\begin{array}{l}
\dot{y}_{1} \\
\dot{y}_{2}
\end{array}\right)=\left(\begin{array}{rr}
a(t) & d(t) \\
b(t) & -a(t)
\end{array}\right)\left(\begin{array}{l}
y_{1} \\
y_{2}
\end{array}\right) .
$$

Suppose that $a(-t)=-a(t), b(-t)=b(t), d(-t)=d(t)$ for any $t \in\left[c_{1}, c_{2}\right]$. If $y$ is a solution of the system (4.49), then the function $z(t)=\left(y_{2}(-t), y_{1}(-t)\right)$ satisfies the conjugate system of (4.49) on the segment $\left[c_{1}, c_{2}\right]$.

Lemma 4.5 is verified by simply substituting the solution $z(t)=\left(y_{2}(-t), y_{1}(-t)\right)$ into the conjugate system.

Proof of Lemma 4.4. Let $\tau \in[0, T]$ be such that

$$
\widetilde{x}_{1}^{\tau}(0)=0 \text {. }
$$

Using conditions (4.46) and (4.47) it is easy to verify that the function $p(t)=$ $\left(-\widetilde{x}_{1}^{\tau}(-t), \widetilde{x}_{2}^{\tau}(-t)\right)$ is a solution of the generating system (1.1). But $p(0)=\widetilde{x}^{\tau}(0)$; consequently,

$$
\left(-\widetilde{x}_{1}^{\tau}(-t), \widetilde{x}_{2}^{\tau}(-t)\right)=\widetilde{x}^{\tau}(t) \quad \text { for any } t \in[0, T] .
$$

Consider the generating system (1.1) linearized on $\widetilde{x}^{\tau}$ :

$$
\left(\begin{array}{c}
\dot{y}_{1} \\
\dot{y}_{2}
\end{array}\right)=\left(\begin{array}{ll}
\left(f_{1}\right)_{(1)}^{\prime}\left(\widetilde{x}^{\tau}(t)\right) & \left(f_{1}\right)_{(2)}^{\prime}\left(\widetilde{x}^{\tau}(t)\right) \\
\left(f_{2}\right)_{(1)}^{\prime}\left(\widetilde{x}^{\tau}(t)\right) & \left(f_{2}\right)_{(2)}^{\prime}\left(\widetilde{x}^{\tau}(t)\right)
\end{array}\right)\left(\begin{array}{c}
y_{1} \\
y_{2}
\end{array}\right) .
$$

It follows from condition (4.48) that

$$
\left(f_{1}\right)_{(1)}^{\prime}\left(\widetilde{x}^{\tau}(t)\right)=-\left(f_{2}\right)_{(2)}^{\prime}\left(\widetilde{x}^{\tau}(t)\right) .
$$

From (4.46) we have $-\left(f_{1}\right)_{(1)}^{\prime}\left(-\xi_{1}, \xi_{2}\right)=\left(f_{1}\right)_{(1)}^{\prime}\left(\xi_{1}, \xi_{2}\right)$ and taking (4.50) into account we obtain

$$
\left(f_{1}\right)_{(1)}^{\prime}\left(\widetilde{x}^{\tau}(t)\right)=-\left(f_{1}\right)_{(1)}^{\prime}\left(\widetilde{x}^{\tau}(-t)\right) .
$$

From (4.47) we have $-\left(f_{2}\right)_{(1)}^{\prime}\left(\xi_{1}, \xi_{2}\right)=\left(f_{2}\right)_{(1)}^{\prime}\left(-\xi_{1}, \xi_{2}\right)$ and using (4.50) we obtain

$$
\left(f_{2}\right)_{(1)}^{\prime}\left(\widetilde{x}^{\tau}(-t)\right)=\left(f_{2}\right)_{(1)}^{\prime}\left(\widetilde{x}^{\tau}(t)\right) .
$$


Finally, from (4.46) we have $\left(f_{1}\right)_{(2)}^{\prime}\left(-\xi_{1}, \xi_{2}\right)=\left(f_{1}\right)_{(2)}^{\prime}\left(\xi_{1}, \xi_{2}\right)$ and taking (4.50) into account we obtain

$$
\left(f_{1}\right)_{(2)}^{\prime}\left(\widetilde{x}^{\tau}(t)\right)=\left(f_{1}\right)_{(2)}^{\prime}\left(\widetilde{x}^{\tau}(-t)\right) .
$$

Thus, the hypotheses of Lemma 4.5 hold, and based on this we see that the functions

$$
\widehat{\widehat{z}}^{\tau}(t)=\left(\begin{array}{c}
\widehat{y}_{2}^{\tau}(-t) \\
\widehat{y}_{1}^{\tau}(-t)
\end{array}\right) \quad \text { and } \quad \widetilde{\widetilde{z}}^{\tau}(t)=\left(\begin{array}{c}
\dot{\widetilde{x}}_{2}^{\tau}(-t) \\
\dot{\widetilde{x}}_{1}^{\tau}(-t)
\end{array}\right)
$$

satisfy the conjugate system of (4.51),

$$
\dot{z}=-\left(f^{\prime}\left(\widetilde{x}^{\tau}(t)\right)\right) z .
$$

From (4.50), for any $t \in[0, T]$ we have

$$
\dot{\vec{x}}_{1}^{\tau}(-t)=\dot{\tilde{x}}_{1}^{\tau}(t), \quad-\dot{\tilde{x}}_{2}^{\tau}(-t)=\dot{\vec{x}}_{2}^{\tau}(t)
$$

We now show that, together with $\widehat{y}^{\tau}$, the function $p(t)=\left(-\widehat{y}_{1}^{\tau}(-t), \widehat{y}_{2}^{\tau}(-t)\right)$ is a solution of the system (4.51). Indeed, from (4.52) and (4.54) we have

$$
\dot{p}_{1}(t)=-\left(f_{1}\right)_{(1)}^{\prime}\left(\widetilde{x}^{\tau}(t)\right) y_{1}^{\tau}(-t)+\left(f_{1}\right)_{(2)}^{\prime}\left(\widetilde{x}^{\tau}(t)\right) y_{2}^{\tau}(-t),
$$

and from (4.53), (4.48), and (4.52) we have

$$
\dot{p}_{2}(t)=-\left(f_{2}\right)_{(1)}^{\prime}\left(\widetilde{x}^{\tau}(t)\right) y_{1}^{\tau}(-t)+\left(f_{2}\right)_{(2)}^{\prime}\left(\widetilde{x}^{\tau}(t)\right) y_{2}^{\tau}(-t) .
$$

At the same time, as $\left\langle\widehat{\widehat{z}}^{0}(0), \widetilde{x}^{0}(0)\right\rangle=0$, by Perron's lemma (see [36] or [10, Sec. III, $\S 12])$ we have $\left\langle\widehat{\widehat{z}}^{\tau}(0), \widetilde{x}^{\tau}(0)\right\rangle=0$, that is,

$$
\left\langle\left(\begin{array}{c}
\widehat{y}_{2}^{\tau}(0) \\
\widehat{y}_{1}^{\tau}(0)
\end{array}\right), \widetilde{x}^{\tau}(0)\right\rangle=0 \text {. }
$$

But $\widetilde{x}_{1}^{\tau}(0)=0$; therefore from the last equality we conclude that $\widehat{y}_{1}^{\tau}(0)=0$. This allows us to assert that $p(0)=\widehat{y}^{\tau}(0)$; therefore,

$$
\left(-\widehat{y}_{1}^{\tau}(-t), \widehat{y}_{2}^{\tau}(-t)\right)=\widehat{y}^{\tau}(t), \quad t \in \mathbb{R} .
$$

Now using (4.56) and (4.57), the functions $\widehat{\widehat{z}}^{\tau}$ and $\widetilde{\widetilde{z}}^{\tau}$ can be rewritten in the form

$$
\widehat{\widehat{z}}^{\tau}(t)=\left(\begin{array}{r}
\widehat{y}_{2}^{\tau}(t) \\
-\widehat{y}_{1}^{\tau}(t)
\end{array}\right) \quad \text { and } \quad \widetilde{\widetilde{z}}^{\tau}(t)=\left(\begin{array}{r}
-\dot{\tilde{x}}_{2}^{\tau}(t) \\
\dot{\tilde{x}}_{1}^{\tau}(t)
\end{array}\right) .
$$

Since the functions $\widehat{\widehat{z}}^{\tau}$ and $\widetilde{\widetilde{z}}^{\tau}$ are solutions of the conjugate system (4.55), the functions $\widehat{\widehat{z}}$ and $\widetilde{\widetilde{z}}$ are solutions of the conjugate system (2.6). But

$$
\widehat{\widehat{z}}(0)=\left(\begin{array}{r}
\widehat{y}_{2}(0) \\
-\widehat{y}_{1}(0)
\end{array}\right)=\frac{1}{\|\dot{\tilde{x}}(0)\|^{2}} \dot{\widetilde{x}}(0)=\widehat{z}(0), \quad \widetilde{\widetilde{z}}(0)=\left(\begin{array}{r}
-\dot{\widetilde{x}}_{2}(0) \\
\dot{\widetilde{x}}_{1}(0)
\end{array}\right)=\widetilde{z}(0) ;
$$

consequently,

$$
\widehat{z}(t)=\left(\begin{array}{r}
\widehat{y}_{2}(t) \\
-\widehat{y}_{1}(t)
\end{array}\right), \quad \widetilde{z}(t)=\left(\begin{array}{r}
-\dot{\widetilde{x}}_{2}(t) \\
\dot{\widetilde{x}}_{1}(t)
\end{array}\right) .
$$

The lemma is proved.

Thus, if the symmetry conditions of Lemma 4.4 hold, it not only reduces the function $M_{E}$ to the classical function but also simplifies the calculation of the function $M_{A}^{s}$. We present one corollary of Lemma 4.2, which can also be used to simplify the calculation of the function $M_{A}^{s}$. 
Corollary 4.1. Suppose that the algebraic multiplicity of the multiplier +1 of the linearized system (1.4) is equal to 2 and $\widehat{z}_{2}(0)=0$. Then

$$
M_{A}^{s}(\theta)=M_{A}^{T}(0)-\frac{\widehat{z}_{2}(T)}{\widetilde{z}_{2}(0)} \int_{s+\theta}^{T}\langle\widetilde{z}(\tau), g(\tau-\theta, \widetilde{x}(\tau))\rangle d \tau .
$$

Proof. Making the change of variables $t=\tau+T$ in the integral and Lemma 4.2 we can carry out the following transformation:

$$
\begin{aligned}
M_{A}^{s}(\theta)= & \int_{s-T+\theta}^{s+\theta}\langle\widehat{z}(\tau), g(\tau-\theta, \widetilde{x}(\tau))\rangle d \tau \\
= & \int_{0}^{s+\theta}\langle\widehat{z}(\tau), g(\tau-\theta, \widetilde{x}(\tau))\rangle d \tau+\int_{s-T+\theta}^{0}\langle\widehat{z}(\tau), g(\tau-\theta, \widetilde{x}(\tau))\rangle d \tau \\
= & \int_{0}^{s+\theta}\langle\widehat{z}(\tau), g(\tau-\theta, \widetilde{x}(\tau))\rangle d \tau+\int_{s+\theta}^{T}\langle\widehat{z}(t-T), g(t-\theta, \widetilde{x}(t))\rangle d t \\
= & \int_{0}^{s+\theta}\langle\widehat{z}(\tau), g(\tau-\theta, \widetilde{x}(\tau))\rangle d \tau \\
& +\int_{s+\theta}^{T}\left\langle\left(\widehat{z}(t)-\frac{\widehat{z}_{2}(T)}{\widetilde{z}_{2}(0)} \widetilde{z}(t)\right), g(t-\theta, \widetilde{x}(t))\right\rangle d t \\
= & \int_{0}^{T}\langle\widehat{z}(\tau), g(\tau-\theta, \widetilde{x}(\tau))\rangle d \tau-\frac{\widehat{z}_{2}(T)}{\widetilde{z}_{2}(0)} \int_{s+\theta}^{T}\langle\widetilde{z}(t), g(t-\theta, \widetilde{x}(t))\rangle d t \\
= & M_{A}^{T}(\theta)-\frac{\widehat{z}_{2}(T)}{\widetilde{z}_{2}(0)} \int_{s+\theta}^{T}\langle\widetilde{z}(t), g(t-\theta, \widetilde{x}(t))\rangle d t .
\end{aligned}
$$

The corollary is proved.

We have completed the comparison of the functions $M_{A}^{s}$ and $M_{E}^{s}$ with Malkin's and Mel'nikov's classical functions $\widetilde{M}_{A}$ and $\widetilde{M}_{E}$, respectively; we will now compare Theorem 2.1 with the corresponding classical theorems. We will use several examples to compare the conclusions obtained from Mel'nikov's theorem with those obtained from Theorem 2.1. It is absolutely clear that if we change the generating systems of the examples we give to appropriate systems admitting a $T$-periodic limit cycle, we can also make a comparison between Theorem 2.1 and Malkin's theorem. For example, the system

$$
\left(\begin{array}{c}
\dot{x}_{1} \\
\dot{x}_{2}
\end{array}\right)=\left(\begin{array}{r}
x_{2}+x_{1}\left(x_{1}^{2}+x_{2}^{2}-1\right) \\
-x_{1}+x_{2}\left(x_{1}^{2}+x_{2}^{2}-1\right)
\end{array}\right)
$$

is a suitable one for us to look at; it admits the unique cycle $\widetilde{x}(\theta)=(\sin (\theta), \cos (\theta))$ and, in particular, it satisfies the hypotheses of Lemma 4.1 (see [14, formula 37]). Just making a detailed comparison with Mel'nikov's theorem here is also justified by the fact that we have recently obtained a generalization of Malkin's theorem, which we stated above, that is true in a space of arbitrary dimension (see [11, Corollary 3.5]). The approach proposed in 11] uses a condition of type $\left(C_{M A}\right)$ and cannot be applied to Mel'nikov's case. The same condition is also assumed in the paper [22] cited above, where a formula of the form (1.5) is established in the corresponding case.

In the first example we show that Theorem 2.1 may sharpen the result of Mel'nikov's theorem; in the second we show that it may establish that at least two $T$-periodic solutions exist close to the cycle $\widetilde{x}$, in certain cases where Mel'nikov's theorem guarantees the existence of at least one cycle.

The comparison is conducted in the case of principal resonance, that is, when the smallest period of the cycle $\widetilde{x}$ coincides with the smallest period of the perturbation 
and, moreover, in its simple subcase where the function $M_{E}$ has exactly two zeros on the interval $[0, T)$. This restriction enables us to apply Theorem 3.1 and makes the comparison as easy and descriptive as possible.

Example 4.1. Suppose that we are given the system

$$
\left(\begin{array}{c}
\dot{x}_{1} \\
\dot{x}_{2}
\end{array}\right)=\left(\begin{array}{c}
x_{2}\left(1-x_{1}^{2}-x_{2}^{2}\right) \\
-x_{1}\left(1-x_{1}^{2}-x_{2}^{2}\right)
\end{array}\right)+\varepsilon\left(\begin{array}{c}
0 \\
\sin (w t)
\end{array}\right) .
$$

For $\varepsilon=0$ the system (4.58) has the form

$$
\left(\begin{array}{l}
\dot{x}_{1} \\
\dot{x}_{2}
\end{array}\right)=\left(\begin{array}{r}
x_{2}\left(1-x_{1}^{2}-x_{2}^{2}\right) \\
-x_{1}\left(1-x_{1}^{2}-x_{2}^{2}\right)
\end{array}\right)
$$

and admits the family of periodic orbits

$$
\left\{\sqrt{1-\alpha}\left(\begin{array}{c}
\sin (\alpha t) \\
\cos (\alpha t)
\end{array}\right)\right\}_{\alpha>0}
$$

We consider the problem of existence of periodic orbits of principal resonance, that is, the problem of perturbing an orbit

$$
\widetilde{x}(t)=\sqrt{1-w}\left(\begin{array}{c}
\sin (w t) \\
\cos (w t)
\end{array}\right)
$$

that has period $T=2 \pi / w$ which coincides with the period of the perturbation. Since

$$
\dot{\widetilde{x}}(t)=w \sqrt{1-w}\left(\begin{array}{r}
\cos (w t) \\
-\sin (w t)
\end{array}\right),
$$

Mel'nikov's function has the form

$$
\widetilde{M}_{E}(\theta)=-\pi \sqrt{1-w} \sin (w \theta) .
$$

Obviously, the function $\widetilde{M}_{E}$ has two simple zeros $\theta_{1}=0$ and $\theta_{2}=\pi / w$ on the interval $[0,2 \pi / w)$, and Mel'nikov's theorem guarantees that for any $w \in(0,1)$ and for sufficiently small $\varepsilon>0$, the system (4.58) has at least two T-periodic solutions $\widetilde{x}_{\varepsilon, 1}$ and $\widetilde{x}_{\varepsilon, 2}$ converging to the cycle $\widetilde{x}$ as $\varepsilon \rightarrow 0$.

Now let us see what result can be obtained using Theorem 2.1

We observe that the generating system (4.59) satisfies the hypotheses of Lemma 4.4 consequently,

$$
M_{E}^{s}(\theta)=\widetilde{M}_{E}(\theta) \quad \text { and } \quad M_{A}^{s}(\theta)=-\int_{s-T}^{s} \operatorname{det}\|(\widehat{y}(\tau), g(\tau-\theta, \widetilde{x}(\tau)))\| d \tau .
$$

The system (4.59) linearized on $\widetilde{x}$ has the form

$$
\left(\begin{array}{l}
\dot{y}_{1} \\
\dot{y}_{2}
\end{array}\right)=\left(\begin{array}{ll}
-2(1-w) \sin (w t) \cos (w t) & w-2(1-w) \cos ^{2}(w t) \\
-w+2(1-w) \sin ^{2}(w t) & 2(1-w) \sin (w t) \cos (w t)
\end{array}\right)\left(\begin{array}{l}
y_{1} \\
y_{2}
\end{array}\right) .
$$

A solution $\widehat{y}$ of the system (4.61) with the initial condition

$$
\widehat{y}(0)=\frac{1}{\|\dot{\tilde{x}}(0)\|^{2}} \dot{\widetilde{x}}(0)^{\perp}=\frac{1}{w \sqrt{1-w}}\left(\begin{array}{l}
0 \\
1
\end{array}\right)
$$

is given by the formula

$$
\widehat{y}(t)=\frac{1}{w \sqrt{1-w}}\left(\begin{array}{r}
-2(1-w) t \cos (w t)+\sin (w t) \\
2(1-w) t \sin (w t)+\cos (w t)
\end{array}\right) .
$$


Therefore,

$$
\begin{aligned}
M_{A}^{s}(0) & =\frac{\pi}{w^{3} \sqrt{1-w}}\left(2(1-w) \sin ^{2}(w s)-1\right), \\
M_{A}^{s}(\pi / w) & =-\frac{\pi}{w^{3} \sqrt{1-w}}\left(2(1-w) \sin ^{2}(w s)-1\right) .
\end{aligned}
$$

Thus, for condition $(A)$ of Theorem 2.1 to hold it is necessary that

$$
w \in\left(\frac{1}{2}, 1\right) .
$$

If the last condition holds, then $M_{A}^{s}(0) M_{A}^{s}(\pi / w)<0$; thus, by Theorem 3.1, in Theorem 2.1 condition $(B)$ on the index also holds. Thus, based on Theorem 2.1] we have the following: for any $w \in(1 / 2,1)$ and for sufficiently small $\varepsilon>0$, the system (4.58) has at least two $(2 \pi / w)$-periodic solutions $\widetilde{x}_{\varepsilon, 1}$ and $\widetilde{x}_{\varepsilon, 2}$ converging to the cycle $\widetilde{x}$ as $\varepsilon \rightarrow 0$. The solution $\widetilde{x}_{\varepsilon, 1}$ is contained strictly inside the cycle $\widetilde{x}$, and the solution $\widetilde{x}_{\varepsilon, 1}$ strictly outside. Other $(2 \pi / w)$-periodic solutions of the system (4.58) for these values of $w$ and $\varepsilon$ also do not intersect the cycle $\widetilde{x}$.

In Example 4.1 we see that the domain of the parameter $w>0$ for which Mel'nikov's method can be applied is wider than the domain for which Theorem 2.1 can be applied, but in this more narrow domain, Theorem 2.1 yields new properties for the periodic solutions of principal resonance.

We now produce an example in which Theorem 2.1 not only yields new properties for the periodic solutions of principal resonance but also proves that more of them exist.

Example 4.2. We adapt the system in the preceding example as follows:

$$
\begin{aligned}
& \dot{x}_{1}=x_{2}\left(1-\frac{1}{5}\left(x_{1}^{2}+x_{2}^{2}\right)\right), \\
& \dot{x}_{2}=-x_{1}\left(1-\frac{1}{5}\left(x_{1}^{2}+x_{2}^{2}\right)\right)+\varepsilon\left(\sin \left(\frac{4}{5} t\right)-x_{1}\right)^{3}+\varepsilon x_{1}
\end{aligned}
$$

and examine the perturbation of the generating orbit,

$$
\widetilde{x}(t)=\left(\begin{array}{c}
\sin \left(\frac{4}{5} t\right) \\
\cos \left(\frac{4}{5} t\right)
\end{array}\right),
$$

with period $T=5 \pi / 2$ coinciding with the period of the perturbation. Mel'nikov's function corresponding to this problem is written as

$$
M_{E}(\theta)=\frac{3 \pi}{4} \sin \left(\frac{8}{5} t\right)-\frac{3 \pi}{2} \sin \left(\frac{4}{5} t\right)
$$

and admits two zeros $\theta_{1}=0$ and $\theta_{2}=5 \pi / 4$. However only the second of them is simple, while the first is cubic, that is, $M^{\prime}(0)=0, M^{\prime \prime}(0)=0$, and $M^{\prime \prime \prime}(0)=-288 \pi / 125$.

Thus, from Mel'nikov's theorem we conclude that for sufficiently small $\varepsilon>0$, the system (4.62) has at least one T-periodic solution $\widetilde{x}_{\varepsilon}$ converging to the cycle $\widetilde{x}$ as $\varepsilon \rightarrow 0$.

It is easy to verify that the system (4.62) with $\varepsilon=0$ linearized on $\widetilde{x}$ coincides with the system (4.61) with $w=4 / 5$. Therefore, taking into accoount the fact that the initial condition of the solution $\widehat{y}$ is given by the formula

$$
\widehat{y}(0)=\frac{1}{\|\dot{\tilde{x}}(0)\|^{2}} \dot{\tilde{x}}(0)^{\perp}=\frac{1}{4 / 5}\left(\begin{array}{l}
0 \\
1
\end{array}\right),
$$


we conclude that

$$
\widehat{y}(t)=\frac{1}{4 / 5}\left(\begin{array}{r}
-\frac{2}{5} t \cos \left(\frac{4}{5} t\right)+\sin \left(\frac{4}{5} t\right) \\
\frac{2}{5} t \sin \left(\frac{4}{5} t\right)+\cos \left(\frac{4}{5} t\right)
\end{array}\right) .
$$

Using Lemma 4.4, or to be specific (4.60), we obtain the following expressions for $M_{A}^{s}(0)$ and $M_{A}^{s}(5 \pi / 4)$ :

$$
\begin{aligned}
M_{A}^{s}(0) & =-\frac{25 \pi}{64} \cos \left(\frac{8}{5} s\right)-\frac{25 \pi}{16} \\
M_{A}^{s}(5 \pi / 4) & =-\frac{25 \pi}{64} \cos \left(\frac{16}{5} s\right)+\frac{75 \pi}{64} \cos \left(\frac{8}{5} s\right)+\frac{125 \pi}{16}
\end{aligned}
$$

and it is easy to verify that $M_{A}^{s}(0)<0$ and $M_{A}^{s}(5 \pi / 4)>0$ for all $s \in[0,5 \pi / 2]$.

Therefore, based on Theorem 2.1, we have the following: for all sufficiently small $\varepsilon>0$, the system (4.62) has at least two T-periodic solutions $\widetilde{x}_{\varepsilon, 1}$ and $\widetilde{x}_{\varepsilon, 2}$ converging to the cycle $\widetilde{x}$ as $\varepsilon \rightarrow 0$. The solution $\widetilde{x}_{\varepsilon, 1}$ lies strictly inside the cycle $\widetilde{x}$, and the solution $\widetilde{x}_{\varepsilon, 1}$ strictly outside.

Thus, the fact that Mel'nikov's function has repeated roots does not undermine the efficacy of Theorem 2.1.

We now turn to the case where the perturbation is not a differentiable function. We point out that the question of the existence of periodic solutions in perturbed systems of type (1.2) in the case of zero or linear generating systems was considered by Mitropol'skiı̌ [29, Samoulenko [40, Mawhin [25, 26], Buică and Llibre [4, and many others. In the following example we demonstrate an application of Theorem 2.1 to systems with a nondifferentiable right-hand side for a nonzero nonlinear generating system. As a perturbation we take the so-called jumping nonlinearity, see [19], and we take Duffing's equation as the generating system.

Example 4.3. Consider the problem of whether Duffing's equation with jumping nonlinearity

$$
\ddot{u}+u+u^{3}=\varepsilon\left(\mu x_{1}^{+}+\nu x_{1}^{-}+\cos ((1+\delta) t)\right)
$$

has resonance periodic solutions.

We establish the following simple lemma.

Lemma 4.6. Consider the system

$$
\begin{aligned}
& \dot{x}_{1}=x_{2}, \\
& \dot{x}_{2}=-x_{1}+\varepsilon\left(\mu x_{1}^{+}+\nu x_{1}^{-}+\cos (t)\right),
\end{aligned}
$$

where $a^{+}:=\max \{a, 0\}$ and $a^{-}:=\max \{-a, 0\}$. Let $\widetilde{\widetilde{x}}(t)=(\sin t, \cos t)$. If $|\mu-\nu| \neq 2$, then the corresponding functions $M_{A}^{s}$ and $M_{E}^{s}$ satisfy condition (A) of Theorem 2.1. But if $|\mu-\nu|<2$, then

$$
\operatorname{ind}(\widetilde{\widetilde{x}}, \Phi) \in\{0,2\}
$$


Proof. We have

$$
\begin{aligned}
M_{A}^{s}(\theta)= & \int_{0}^{2 \pi} \sin \tau\left(\mu \widetilde{x}_{1}^{+}(\tau)+\nu \widetilde{x}_{1}^{-}(\tau)+\cos (\tau-\theta)\right) d \tau \\
= & \mu \int_{0}^{\pi} \sin \tau \sin \tau d \tau-\nu \int_{\pi}^{2 \pi} \sin \tau \sin \tau d \tau \\
& \quad+\int_{0}^{2 \pi} \sin \tau \cos \tau d \tau \cos \theta+\int_{0}^{2 \pi} \sin \tau \sin \tau d \tau \sin \theta \\
= & \mu \frac{\pi}{2}-\nu \frac{\pi}{2}+\pi \sin \theta, M_{E}^{s}(\theta) \\
= & \int_{0}^{2 \pi} \cos \tau\left(\mu \widetilde{x}_{1}^{+}(\tau)+\nu \widetilde{x}_{1}^{-}(\tau)+\cos (\tau-\theta)\right) d \tau \\
= & \mu \int_{0}^{\pi} \cos \tau \sin \tau d \tau-\nu \int_{\pi}^{2 \pi} \cos \tau \sin \tau d \tau \\
& \quad+\int_{0}^{2 \pi} \cos \tau \cos \tau d \tau \cos \theta+\int_{0}^{2 \pi} \cos \tau \sin \tau d \tau \sin \theta=\pi \cos \theta
\end{aligned}
$$

Since $|\mu-\nu|<2$ by the hypothesis of the lemma, $\theta_{0}=\arcsin \frac{-\mu+\nu}{2}$ is the only zero of the function $M_{E}$ on the interval $[-\pi / 2, \pi / 2]$. Therefore on the interval $[0,2 \pi)$ the function $M_{E}$ has exactly two roots:

$$
\begin{aligned}
& \theta_{1}= \begin{cases}\theta_{1}=\theta_{0} & \text { if } \theta_{0} \geq 0, \\
\theta_{1}=\theta_{0}+\pi & \text { otherwise, }\end{cases} \\
& \theta_{2}=\theta_{1}+\pi .
\end{aligned}
$$

The function $M_{E}$ has exactly two zeros, $\theta_{1}=\pi / 2$ and $\theta_{2}=3 \pi / 2$ on the interval $[0,2 \pi)$. At these points we have

$$
M_{A}(\pi / 2)=\mu \frac{\pi}{2}-\nu \frac{\pi}{2}+\pi, \quad M_{A}(3 \pi / 2)=\mu \frac{\pi}{2}-\nu \frac{\pi}{2}-\pi .
$$

Thus, if $|\mu-\nu| \neq 2$, then

$$
M_{A}(\pi / 2) \neq 0, \quad M_{A}(3 \pi / 2) \neq 0 .
$$

But if $|\mu-\nu|<2$, then

$$
M_{A}(\pi / 2)>0, \quad M_{A}(3 \pi / 2)<0
$$

and by Theorem 3.1 we have ind $(\widetilde{\widetilde{x}}, \Phi) \in\{0,2\}$.

The lemma is proved.

Thus, we return to the system (4.63). We denote by $u_{\delta}$ the unique periodic orbit of the generating equation

$$
\ddot{u}+u+u^{3}=0
$$

up to a shift, with the smallest period $\frac{2 \pi}{1+\delta}$. If $u$ is a solution of equation (4.63), then $v=(u, \dot{u})$ satisfies the system

$$
\begin{aligned}
& \dot{v}_{1}=v_{2}, \\
& \dot{v}_{2}=-v_{1}-v_{1}^{3}+\varepsilon\left(\mu v_{1}^{+}+\nu v_{1}^{-}+\cos ((1+\delta) t)\right) ;
\end{aligned}
$$

conversely, if $v$ is a solution of the system (4.65), then $v_{1}$ is a solution of the system (4.63). Without loss of generality, in the result we give below we can assume that $\dot{u}_{\delta}(0)=0$ and 
$u_{\delta}(0)>0$. Making the change of variables

$$
x(t)=\frac{v(t)}{u_{\delta}(0)},
$$

we pass from the system (4.65) to the system

$$
\begin{aligned}
& \dot{x}_{1}=x_{2}, \\
& \dot{x}_{2}=-x_{1}-\left(u_{\delta}(0)\right)^{2} x_{1}^{3}+\varepsilon \frac{1}{u_{\delta}(0)} \cos ((1+\delta) t)+\varepsilon\left(\mu x_{1}^{+}+\nu x_{1}^{-}\right) .
\end{aligned}
$$

Suppose that $|\mu-\nu|<2$. We now establish that there exists $\delta_{0}>0$ such that for $\delta \in\left(0, \delta_{0}\right]$ the hypotheses of Theorem 2.1 related to the functions $M_{E}^{s}, M_{A}^{s}$, and $\Phi$, hold for the system (4.66) with $\widetilde{x}(t)=\frac{v_{\delta}(t)}{u_{\delta}(0)}$ and $T=\frac{2 \pi}{1+\delta}$. For this to hold, in turn, it is sufficient to establish a similar result for the system

$$
\begin{aligned}
& \dot{x}_{1}=x_{2}, \\
& \dot{x}_{2}=-x_{1}-\left(u_{\delta}(0)\right)^{2} x_{1}^{3}+\varepsilon \cos ((1+\delta) t)+\varepsilon\left(\mu x_{1}^{+}+\nu x_{1}^{-}\right) .
\end{aligned}
$$

The period of the orbits of the generating system

$$
\dot{x}_{1}=x_{2}, \quad \dot{x}_{2}=-x_{1}-\left(u_{\delta}(0)\right)^{2} x_{1}^{3}
$$

varies monotonically from $2 \pi$ to 0 as the initial condition of the orbit varies from $(0,0)$ to $(+\infty, 0)$ (see, for example, 9]). Consequently, $u_{\delta}(0) \rightarrow 0$ as $\delta \rightarrow 0$. But for $\delta=0$ the fact that the desired result holds for the system (4.67) follows from Lemma 4.6. consequently, this assertion also remains valid for small $\delta>0$.

Thus, we have established that if $|\mu-\nu|<2$, then there exists $\delta_{0}>0$ such that to each $\delta \in\left[0, \delta_{0}\right]$ there corresponds $\varepsilon_{0}>0$ with the following properties:

1) for each $\varepsilon \in\left(0, \varepsilon_{0}\right]$, the perturbed Duffing equation (4.63) has at least two $\frac{2 \pi}{1+\delta}$ periodic solutions $\widetilde{u}_{\delta, \varepsilon}, \widetilde{\widetilde{u}}_{\delta, \varepsilon}$ such that the curve $t \rightarrow\left(\widetilde{u}_{\delta, \varepsilon}(t), \dot{\tilde{u}}_{\delta, \varepsilon}(t)\right)$ lies strictly inside the curve $t \rightarrow\left(u_{\delta}(t), \dot{u}_{\delta}(t)\right)$, while the curve $t \rightarrow\left(\widetilde{\widetilde{u}}_{\delta, \varepsilon}(t), \dot{\widetilde{\widetilde{u}}}_{\delta, \varepsilon}(t)\right)$ lies strictly outside it;

2) for every $\frac{2 \pi}{1+\delta}$-periodic solution $u$ of the system (4.63) with $\varepsilon \in\left(0, \varepsilon_{0}\right]$, the curve $t \rightarrow(u(t), \dot{u}(t))$ has no intersection points with the curve $t \rightarrow\left(u_{\delta}(t), \dot{u}_{\delta}(t)\right)$;

3) the solutions $\widetilde{u}_{\delta, \varepsilon}$ and $\widetilde{\widetilde{u}}_{\delta, \varepsilon}$ satisfy the condition

$$
\widetilde{u}_{\delta, \varepsilon}(t) \rightarrow u_{\delta}(t-\tilde{\theta}) \quad \text { and } \quad \widetilde{\widetilde{u}}_{\delta, \varepsilon}(t) \rightarrow u_{\delta}(t-\widetilde{\widetilde{\theta}}) \quad \text { as } \varepsilon \rightarrow 0
$$

for some $\widetilde{\theta}, \widetilde{\widetilde{\theta}} \in\left[0, \frac{2 \pi}{1+\delta}\right]$.

If $\nu=\mu=0$, then the result we have obtained is an addition to the known results on the qualitative behaviour of periodic trajectories of the Duffing equation; see, for example, Morozov's paper [31.

\section{Degenerate Resonances. A COMParison with Yagasaki's theorem}

In this section we consider the case where the $T$-periodic cycle $\widetilde{x}$ is degenerate, that is, all the solutions of the linearized system (1.4) are $T$-periodic. The $T$-periodic solutions of the perturbed system generated by such cycles are called degenerate resonances. If $\widetilde{x}$ is embedded into a family of cycles $\left\{\widetilde{x}_{\alpha}\right\}_{\alpha>0}$ of the autonomous system with periods $T(\alpha)$, that is, if

$$
\widetilde{x}=\widetilde{x}_{\alpha_{0}}
$$


for some $\alpha_{0}>0$, then, as the following lemma shows, the assumption that there is degeneracy almost always holds in the case where $T\left(\alpha_{0}\right)$ is a critical period, that is,

$$
T^{\prime}\left(\alpha_{0}\right)=0 .
$$

We note that the problem of the existence of critical periods for cycles embedded in a family of cycles of an autonomous system has been studied intensively; see [6, 7, 8, 43].

Without loss of generality, in what follows we can assume that

$$
\widetilde{x}_{\alpha}(0)=\left(\begin{array}{c}
0 \\
J(\alpha)
\end{array}\right) .
$$

Lemma 5.1. If the conditions $T^{\prime}\left(\alpha_{0}\right)=0, J^{\prime}\left(\alpha_{0}\right) \neq 0$ hold for the orbit $\widetilde{x}_{\alpha_{0}}$ and the first component of the vector $\dot{\widetilde{x}}_{\alpha_{0}}(0)$ is nonzero, then each solution of the linearized system (1.4) with $\widetilde{x}=\widetilde{x}_{\alpha_{0}}$ is $T\left(\alpha_{0}\right)$-periodic.

Proof. It will be convenient for us to use the following notation:

$$
x(t, \alpha):=\widetilde{x}_{\alpha}(t) .
$$

Since the right-hand side of the generating system (1.1) is continuously differentiable, it follows (see, for example, Pontryagin's book [37, Ch. 4, §24, Theorem 17]) that the function $(t, \alpha) \rightarrow x(t, \alpha)$ is continuously differentiable with respect to all its variables. By differentiating the identity

$$
x_{t}^{\prime}(t, \alpha)=f(x(t, \alpha))
$$

with respect to $\alpha$ we obtain

$$
x_{t \alpha}^{\prime \prime}(t, \alpha)=f^{\prime}(x(t, \alpha)) x_{\alpha}^{\prime}
$$

consequently, $\widetilde{y}=x_{\alpha}^{\prime}\left(\cdot, \alpha_{0}\right)$ is a solution of the linearized system (1.4) with $\widetilde{x}=x\left(\cdot, \alpha_{0}\right)$. We have

$$
\begin{aligned}
\widetilde{y}\left(T\left(\alpha_{0}\right)\right)-\widetilde{y}(0)= & \lim _{\Delta \rightarrow 0} \frac{x\left(T\left(\alpha_{0}\right), \alpha_{0}+\Delta\right)-x\left(T\left(\alpha_{0}\right), \alpha_{0}\right)}{\Delta} \\
& -\lim _{\Delta \rightarrow 0} \frac{x\left(0, \alpha_{0}+\Delta\right)-x\left(0, \alpha_{0}\right)}{\Delta} \\
= & \lim _{\Delta \rightarrow 0} \frac{x\left(T\left(\alpha_{0}\right), \alpha_{0}+\Delta\right)-x\left(0, \alpha_{0}+\Delta\right)-x\left(T\left(\alpha_{0}\right), \alpha_{0}\right)+x\left(0, \alpha_{0}\right)}{\Delta} \\
= & \lim _{\Delta \rightarrow 0} \frac{x\left(T\left(\alpha_{0}\right), \alpha_{0}+\Delta\right)-x\left(0, \alpha_{0}+\Delta\right)}{\Delta} \\
= & \left(x\left(T(\cdot), \alpha_{0}\right)\right)^{\prime}\left(\alpha_{0}\right)=x_{t}^{\prime}\left(T\left(\alpha_{0}\right), \alpha_{0}\right) T^{\prime}\left(\alpha_{0}\right)=0 ;
\end{aligned}
$$

that is, $\widetilde{y}$ is a $T\left(\alpha_{0}\right)$-periodic solution of the system (1.4). But

$$
x(0, \alpha)=\left(\begin{array}{c}
0 \\
J(\alpha)
\end{array}\right)
$$

consequently,

$$
\widetilde{y}(0)=x_{\alpha}^{\prime}\left(0, \alpha_{0}\right)=\left(\begin{array}{c}
0 \\
J^{\prime}\left(\alpha_{0}\right)
\end{array}\right) .
$$

At the same time, according to the hypotheses of the lemma, the first component of the vector $\dot{\widetilde{x}}_{\alpha_{0}}(0)$ is nonzero; therefore $\widetilde{y}$ and $x_{t}^{\prime}\left(\cdot, \alpha_{0}\right)$ are two linearly independent $T\left(\alpha_{0}\right)$ periodic solutions of the (two-dimensional) system (1.4), which obviously implies the desired result.

The lemma is proved. 
Lemma 5.1 is a criterion for the degeneracy of a cycle embedded into a family of cycles. However, for a cycle to be degenerate it is not at all necessary that it be embedded into a family of cycles; isolated cycles can also be degenerate. Generally speaking, to study the existence of degenerate resonances in the perturbed system (1.2) in this latter case we could use the general Rhouma-Chicone theorem ([39, Theorem 4.1]), but it only works in the case where the perturbation depends on the phase variable (see [39, formula 2.7]), which we do not require in the theorems presented below.

If the cycle $\widetilde{x}$ is degenerate, then the functions $M_{A}^{s}$ and $M_{E}^{s}$ are obviously independent of $s$ and Theorem 2.1 takes the following form.

Theorem 5.1. Suppose that the degenerate cycle $\widetilde{x}$ satisfies condition $(C)$ and the perturbation is continuous. Suppose that for any $\theta_{0} \in[0, T]$ such that $M_{E}\left(\theta_{0}\right)=0$ we have

$$
M_{A}\left(\theta_{0}\right) \neq 0 \text {. }
$$

Then for sufficiently small $\varepsilon>0$ every T-periodic solution $\widetilde{x}_{\varepsilon}$ of the perturbed system (1.2) must satisfy

$$
\widetilde{x}_{\varepsilon}(t) \neq \widetilde{x}(s) \quad \text { for all } t, s \in[0, T] .
$$

If in addition

$$
\operatorname{ind}(\widetilde{x}, \Phi) \neq 1 \text {, }
$$

then for all sufficiently small $\varepsilon>0$ the perturbed system (1.2) must have at least two $T$ periodic solutions $\widetilde{x}_{\varepsilon, 1}$ and $\widetilde{x}_{\varepsilon, 2}$ satisfying (5.69). Both solutions converge to $\widetilde{x}$ as $\varepsilon \rightarrow 0$. Furthermore, the solution $\widetilde{x}_{\varepsilon, 1}$ lies inside the cycle $\widetilde{x}$, and the solution $\widetilde{x}_{\varepsilon, 2}$ lies outside it.

The hypotheses of Theorem 2.1 take a very simple form if in addition to the degeneracy it is known that the function $M_{E}$ has exactly two zeros on $[0, T)$. For this reason we state the corresponding assertion as a separate theorem.

Theorem 5.2. Suppose that the degenerate cycle $\widetilde{x}$ satisfies condition $(C)$ and the perturbation is continuous. Suppose that the function $M_{E}$ has exactly two zeros $\theta_{1}$ and $\theta_{2}$ on the interval $[0, T)$ and

$$
M_{A}\left(\theta_{1}\right) \cdot M_{A}\left(\theta_{2}\right) \neq 0 \text {. }
$$

Then for sufficiently small $\varepsilon>0$, every T-periodic solution $\widetilde{x}_{\varepsilon}$ of the perturbed system (1.2) must satisfy condition (5.69). If in addition it is known that

$$
M_{A}\left(\theta_{1}\right) \cdot M_{A}\left(\theta_{2}\right)<0,
$$

then for all sufficiently small $\varepsilon>0$ the perturbed system (1.2) has at least two T-periodic solutions $\widetilde{x}_{\varepsilon, 1}$ and $\widetilde{x}_{\varepsilon, 2}$ satisfying (5.69). Both solutions converge to $\widetilde{x}$ as $\varepsilon \rightarrow 0$. Furthermore, the solution $\widetilde{x}_{\varepsilon, 1}$ lies inside the cycle $\widetilde{x}$, and the solution $\widetilde{x}_{\varepsilon, 2}$ outside.

Clearly, the theorem obtained from Theorem 5.2 by interchanging $M_{E}$ and $M_{A}$ is also true.

As far as the author knows, only the case where a degenerate cycle is embedded in a family of cycles and has critical period has been studied in the literature. Therefore, in order to test Theorem 5.2, this is what we will assume in what follows. In this case the conditions which relate to applying the implicit function theorem become more complicated and depend essentially on the multiplicity of the zero $\alpha_{0}$ for the function $T$. For example, Yagasaki 42 considered the case where

$$
T^{\prime}\left(\alpha_{0}\right)=0, \quad T^{\prime \prime}\left(\alpha_{0}\right) \neq 0 .
$$

Apart from the condition that Mel'nikov's function have a simple root $\theta_{0}$, in Yagasaki's paper it was required that a certain auxiliary function $N$, whose formula contains a 
double integral, have a certain sign at the point $\theta_{0}$. Yagasaki illustrates his theorem by several examples; we choose a modification of one of them ([42, Example in $\S 6.4]$ ) to compare his theorem with Theorem 5.2

Example 5.1. We consider the system

$$
\begin{aligned}
& \dot{x}_{1}=x_{2}\left(\frac{1}{4}\left(x_{1}^{2}+x_{2}^{2}-2\right)^{p}+1\right), \\
& \dot{x}_{2}=-x_{1}\left(\frac{1}{4}\left(x_{1}^{2}+x_{2}^{2}-2\right)^{p}+1\right)+\varepsilon \sin (t) .
\end{aligned}
$$

The generating system admits the family of cycles

$$
\widetilde{x}_{\alpha}(t)=\left(\begin{array}{c}
\alpha \sin \left(\frac{2 \pi}{T(\alpha)} t\right) \\
\alpha \cos \left(\frac{2 \pi}{T(\alpha)} t\right)
\end{array}\right)
$$

with periods

$$
T(\alpha)=\frac{2 \pi}{\frac{1}{4}\left(\alpha^{2}-2\right)^{p}+1} .
$$

We examine the perturbation of the cycle corresponding to $\alpha=\sqrt{2}$, that is, of the cycle

$$
\widetilde{x}(t)=\left(\begin{array}{c}
\sqrt{2} \sin t \\
\sqrt{2} \cos t
\end{array}\right)
$$

for which

$$
T(\sqrt{2})=2 \pi, \quad T^{\prime}(\sqrt{2})=\cdots=T^{(p-1)}(\sqrt{2})=0, \quad T^{(p)}(\sqrt{2}) \neq 0 .
$$

In the case $p=2$, Yagasaki calculated the aforementioned function $N$ for the system (5.70) and proved the following assertion (see [42, Theorem 6.4]): For all sufficiently small $\varepsilon>0$ the system (5.70) has at least two T-periodic solutions $\widetilde{x}_{\varepsilon, 1}$ and $\widetilde{x}_{\varepsilon, 2}$ converging to $\widetilde{x}$ as $\varepsilon \rightarrow 0$.

Since for $p \neq 2$ the critical period $2 \pi$ is not double, Yagasaki's result does not apply.

We will try to use Theorem 5.2 . For any positive integer $p$ the generating system linearized on the cycle $\widetilde{x}$ has the form

$$
\left(\begin{array}{l}
\dot{y}_{1} \\
\dot{y}_{2}
\end{array}\right)=\left(\begin{array}{rr}
0 & 1 \\
-1 & 0
\end{array}\right)\left(\begin{array}{l}
y_{1} \\
y_{2}
\end{array}\right)
$$

and, consequently,

$$
\widehat{y}(t)=\frac{1}{\sqrt{2}}\left(\begin{array}{c}
\sin t \\
\cos t
\end{array}\right) .
$$

Thus, for any positive integer $p$, we have

$$
M_{E}(\theta)=-\sqrt{2} \pi \sin (\theta), \quad M_{A}(\theta)=-\frac{1}{\sqrt{2}} \pi \cos (\theta) .
$$

The function $M$ has two zeros, $\theta_{1}=0$ and $\theta_{2}=\pi$, on the interval $[0, T)$; furthermore, $M_{A}(0) \cdot M_{A}(\pi)<0$. Thus, the hypotheses of Theorem 5.2 hold, and so we obtain the following result: Let $p \in \mathbb{N}$ be an arbitrary number. For all sufficiently small $\varepsilon>0$ the system (5.70) has at least two T-periodic solutions $\widetilde{x}_{\varepsilon, 1}$ and $\widetilde{x}_{\varepsilon, 2}$ converging to $\widetilde{x}$ as $\varepsilon \rightarrow 0$. The solutions $\widetilde{x}_{\varepsilon, 1}$ are contained strictly inside the cycle $\widetilde{x}$, and the solutions $\widetilde{x}_{\varepsilon, 1}$ are strictly outside.

Thus, for $p=2$ the result obtained from Theorem 5.2 refines Yagasaki's result and gives exactly the same conclusion for any other multiplicity of the degeneration, where 
his result does not apply. At the same time, it is worth mentioning that Yagasaki's theorem ([42, Theorem 6.4]) guarantees the existence of at least four periodic solutions for some systems (for $p=2$ ), while Theorem 5.2 always guarantees the existence of at least two.

To conclude the section we point out that the behaviour of the trajectories of a perturbed Hamiltonian system near a cycle with critical period was studied in [30] and 32 .

\section{The Location of Stable AND Unstable PERIOdic SOlutions}

As the theorems of Malkin, Mel'nikov and Yagasaki are based on the implicit function theorem, they also provide information about the stability of periodic solutions of the perturbed system (1.2) born from the cycle $\widetilde{x}$. In this section we show that in some cases the knowledge of the index ind $(\widetilde{x}, \Phi)$ allows us to say on which side of the cycle there is at least one stable solution, and on which side at least one unstable solution.

Suppose that

$\left(A_{\mathcal{P}}\right)$ the right-hand sides of the perturbed systems (1.2) are continuously differentiable and the solution $x=: \Omega_{\varepsilon}\left(\cdot, t_{0}, \xi\right)$ of the system (1.2) with the initial condition $x\left(t_{0}\right)=\xi$ can be extended to the segment $[0, T]$ for any $t_{0} \in[0, T], \xi \in \mathbb{R}^{2}$, and $\varepsilon>0$.

In this case, for any $\varepsilon>0$, the Poincaré-Andronov operator $\mathcal{P}_{\varepsilon}=\Omega_{\varepsilon}(T, 0, \cdot)$ for the system (1.2), which corresponds to the problem of finding $T$-periodic solutions for (1.2), is defined.

Theorem 6.1. Suppose that the perturbation in (1.2) is continuous. Suppose that condition (A) of Theorem 2.1 and condition $\left(A_{\mathcal{P}}\right)$ hold. Then there exists $\varepsilon_{0}>0$ such that

$$
\widetilde{x}(\theta) \neq \mathcal{P}_{\varepsilon}(\widetilde{x}(\theta)) \quad \text { for all } \theta \in[0, T], \varepsilon \in\left(0, \varepsilon_{0}\right]
$$

and

$$
\operatorname{ind}\left(\widetilde{x}, I-\mathcal{P}_{\varepsilon}\right)=\operatorname{ind}(\widetilde{x}, \Phi), \quad \varepsilon \in\left(0, \varepsilon_{0}\right] .
$$

Proof. Let $Q_{\varepsilon}$ be the integral operator corresponding to the problem of $T$-periodic solutions for the perturbed system (1.2) and defined by formula (2.19). We denote by $U \subset \mathbb{R}^{2}$ the interior of the cycle $\widetilde{x}$ and set

$$
W_{\varepsilon}=\left\{\widehat{x} \in C\left([0, T], \mathbb{R}^{2}\right): \Omega_{\varepsilon}(0, t, \widehat{x}(t)) \in U \text { for any } t \in[0, T]\right\} .
$$

We claim that there exists $\varepsilon_{0}>0$ such that for any $\varepsilon \in\left(0, \varepsilon_{0}\right]$ and any $\alpha \in\left[0, \varepsilon_{0}\right]$ the following holds:

$$
\text { if } Q_{\varepsilon} x=x \text { and } x \in \bar{W}_{\alpha} \text {, then } x \in W_{0} .
$$

Suppose the opposite; consequently there exist sequences

$$
\begin{gathered}
\left\{\varepsilon_{n}\right\}_{n \in \mathbb{N}} \subset\left(0, \varepsilon_{0}\right], \quad \varepsilon_{n} \rightarrow 0 \quad \text { as } n \rightarrow \infty, \\
\left\{\alpha_{n}\right\}_{n \in \mathbb{N}} \subset\left(0, \varepsilon_{0}\right], \quad\left\{x_{n}\right\}_{n \in \mathbb{N}} \subset C\left([0, T], \mathbb{R}^{2}\right), \quad x_{n} \in \bar{W}_{\alpha_{n}},
\end{gathered}
$$

such that $Q_{\varepsilon_{n}} x_{n}=x_{n}$ and $x_{n} \notin W_{0}$. Since $x_{n} \in \bar{W}_{\varepsilon_{n}}$, we have $x_{n}(0) \in U$. On the other hand, from the relation $x_{n} \notin W_{0}$ we conclude that for any $n \in \mathbb{N}$ there exists $t_{n} \in(0, T]$ such that $x_{n}\left(t_{n}\right) \in \partial U$, which contradicts assertion 1) of Theorem 2.2 .

From (6.71) and assertion 1$)$ of Theorem 2.2 we conclude that the degree $d\left(I-Q_{\varepsilon}, W_{\alpha}\right)$ is defined for any $\alpha \in\left(0, \varepsilon_{0}\right]$ and

$$
d\left(I-Q_{\varepsilon}, W_{\varepsilon}\right)=d\left(I-Q_{\varepsilon}, W_{0}\right), \quad \varepsilon \in\left(0, \varepsilon_{0}\right] .
$$


It follows from the relatedness principle (see [16, Theorem 28.5]) that

$$
d\left(I-Q_{\varepsilon}, W_{\varepsilon}\right)=\operatorname{ind}\left(\widetilde{x}, I-\mathcal{P}_{\varepsilon}\right) .
$$

On the other hand, by assertion 2) of Theorem 2.2 we have

$$
d\left(I-Q_{\varepsilon}, W_{0}\right)=\operatorname{ind}(\widetilde{x}, \Phi) .
$$

The theorem is proved.

We also need the following lemma established by Capietto, Mawhin, and Zanolin.

Capietto-Mawhin-Zanolin Lemma (see [5, Corollary 2]). Suppose that condition $(C)$ holds and the perturbation is continuous. Suppose that condition $\left(A_{\mathcal{P}}\right)$ holds. Then any $T$-periodic function $\widehat{x}$ that is sufficiently close to the cycle $\widetilde{x}$ and does not intersect it satisfies the equality

$$
\operatorname{ind}\left(\widehat{x}, I-\mathcal{P}_{0}\right)=1 .
$$

The proof of the Capietto-Mawhin-Zanolin lemma uses the Kupka-Smale theorem (see [35, Ch. 3]).

Thus, by using the index $\operatorname{ind}(\widetilde{x}, \Phi)$, we have the following information about the location of stable and unstable $T$-periodic solutions of the perturbed system (1.2) near the generating cycle $\widetilde{x}$.

Theorem 6.2. Suppose that the hypotheses of Theorem 6.1 hold, as does condition $(C)$. Choose arbitrary T-periodic functions $\widetilde{x}^{-}$and $\widetilde{x}^{+}$such that $\widetilde{x}^{-}$is contained inside the cycle $\widetilde{x}$, and $\widetilde{x}^{+}$lies outside it, and in the domain $V^{-}$confined between $\widetilde{x}^{-}$and $\widetilde{x}$, and also in the domain $V^{+}$confined between $\widetilde{x}$ and $\widetilde{x}^{+}$, there are no T-periodic solutions of the generating system (1.1). Suppose that in addition it is known that all the fixed points of the Poincaré-Andronov operator $\mathcal{P}_{\varepsilon}$ in $V^{-}$and $V^{+}$are simple. If $\operatorname{ind}(\widetilde{x}, \Phi)>1$ (or $\operatorname{ind}(\widetilde{x}, \Phi)<1)$, then the set $V^{+}\left(V^{-}\right)$contains at least $\mu=|\operatorname{ind}(\widetilde{x}, \Phi)-1|$ fixed points of the operator $\mathcal{P}_{\varepsilon}$ that are saddles, and the set $V^{-}$(respectively, $V^{+}$) contains at least $\mu$ fixed points of the operator $\mathcal{P}_{\varepsilon}$, each of which is either a node or a focus.

We note that the choice of the cycles $\widetilde{x}^{-}$and $\widetilde{x}^{+}$mentioned in the statement is possible by assumption $(C)$.

Proof. Suppose that ind $(\widetilde{x}, \Phi)>1$. Then, by Theorem 6.1 and the Capietto-MawhinZanolin theorem, we have

$$
d\left(\Phi, V^{-}\right)>\mu \quad \text { and } \quad d\left(\Phi, V^{+}\right)<\mu .
$$

Consequently (see [2, Ch. V, $\S 11$, Theorem 26 and Lemma 1]), there exist at least $\mu$ points $\xi_{1}^{-}, \ldots, \xi_{\mu}^{-}$,

$$
\xi_{i}^{-} \in V^{-} \quad \text { for any } i \in\{1, \ldots, \mu\},
$$

and at least $\mu$ points $\xi_{1}^{+}, \ldots, \xi_{\mu}^{+}$,

$$
\xi_{i}^{+} \in V^{+} \quad \text { for any } i \in\{1, \ldots, \mu\} \text {, }
$$

such that

$$
\operatorname{ind}\left(\xi_{i}^{-}\right)=+1 \quad \text { and } \quad \operatorname{ind}\left(\xi_{i}^{+}\right)=-1 \quad \text { for any } i \in\{1, \ldots, \mu\},
$$

that is (see 2, Ch. V, $\S 11$, Theorem 30]), each of the points $\xi_{i}^{-}, i \in\{1, \ldots, \mu\}$, is a node or a focus, and each of the points $\xi_{i}^{+}, i \in\{1, \ldots, \mu\}$ is a saddle.

The case where $\operatorname{ind}(\widetilde{x}, \Phi)<1$ is considered in a similar fashion.

The theorem is proved. 
Conditions to guarantee that the fixed points considered in Theorem 6.2 be simple were proposed in [33] and [41; they made essential use of the differentiability of the right-hand sides of the system (1.2), which was assumed to be in $\left(A_{\mathcal{P}}\right)$. A development of Theorem 6.2 may be obtained based on [34].

\section{Conclusion}

Thus, in the paper, for the study of the problem of birth of $T$-periodic solutions of the perturbed system (1.2) from the $T$-periodic cycle $\widetilde{x}$ of the generating system (1.1), a new characteristic of the generating cycle, ind $(\widetilde{x}, \Phi)$, is proposed. Conditions are given under which the property ind $(\widetilde{x}, \Phi) \neq 1$ implies that the cycle $\widetilde{x}$ generates at least two $T$-periodic solutions of the perturbed system (1.2) lying on different sides of $\widetilde{x}$. Certain conclusions are drawn about on which side of $\widetilde{x}$ do the stable and unstable $T$-periodic solutions that are born lie, depending on whether ind $(\widetilde{x}, \Phi)>1$ or $\operatorname{ind}(\widetilde{x}, \Phi)<1$.

\section{REFERENCES}

[1] P. S. Alexandrov, Combinatorial topology, Gostekhizdat, Moscow, 1947; English transl., Vols. 1, 2, 3, Dover, Mineola, NY, 1998. MR1643155 (99g:55001)

[2] A. A. Andronov, E. A. Leontovich, I. I. Gordon, and A. G. Maier, Qualitative theory of secondorder dynamic systems, Nauka, Moscow, 1966; English transl., Halsted Press (division of John Wiley), New York-Toronto; Israel Program for Scientific Translations, Jerusalem-London, 1973. MR0350126 (50:2619)

[3] K. Borsuk, Drei Sätze über die n-dimensionale euklidische Sphäre, Fund. Math. 20 (1933), 177-190.

[4] A. Buică and J. Llibre, Averaging methods for finding periodic orbits via Brouwer degree, Bull. Sci. Math. 128 (2004), 7-22. MR2033097(2004j:34102)

[5] A. Capietto, J. Mawhin, and F. Zanolin, A continuation theorem for periodic boundary value problems with oscillatory nonlinearities, Nonlinear Differential Equations Appl. 2 (1995), 133-163. MR 1328574 (96a:34034)

[6] C. Chicone and M. Jacobs, Bifurcation of critical periods for plane vector fields, Trans. Amer. Math. Soc. 312 (1989), 433-486. MR 930075 (89h:58139)

[7] S.-N. Chow and J. A. Sanders, On the number of critical points of the period, J. Differential Equations 64 (1986), 51-66. MR849664 (87j:34075)

[8] L. Gavrilov, Remark on the number of critical points of the period, J. Differential Equations 101 (1993), 58-65. MR1199482 (93m:58091)

[9] J. Guckenheimer and P. Holmes, Nonlinear oscillations, dynamical systems, and bifurcations of vector fields, Applied Mathematical Sciences, vol. 42, Springer-Verlag, New York, 1990. MR1139515 (93e:58046)

[10] B. P. Demidovich, Lectures on mathematical stability theory, Moscow University, Moscow, 1998. (Russian)

[11] M. Kamenskii, O. Makarenkov, and P. Nistri, A continuation principle for a class of periodically perturbed autonomous systems, Math. Nachr. 281 (2008), 42-61. MR2376467 (2009a:34077)

[12] M. Kamenskii, V. Obukhovskii, and P. Zecca, Condensing multivalued maps and semilinear differential inclusions in Banach spaces, de Gruyter Series in Nonlinear Analysis and Applications, vol. 7, de Gruyter, Berlin, 2001. MR.1831201 (2002e:47066)

[13] M. Kamenskii, O. Makarenkov, and P. Nistri, Small parameter perturbations of nonlinear periodic systems, Nonlinearity 17 (2004), 193-205. MR2023439 (2004m:34093)

[14] M. Kamenskii, O. Makarenkov, and P. Nistri, Periodic solutions for a class of singularly perturbed systems, Dyn. Contin. Discrete Impuls. Syst. Ser. A Math. Anal. 11 (2004), 41-55. MR2033323 (2005a:34063)

[15] M. Kamenskii, O. Makarenkov, and P. Nistri, An approach to the theory of ordinary differential equations with a small parameter, Dokl. Akad. Nauk 388 (2003), 439-442; English transl., Dokl. Math. 67 (2003), 36-38. MR2004140 (2004g:34079)

[16] M. A. Krasnosel'skiı̌ and P. P. Zabreı̌ko, Geometrical methods of nonlinear analysis, Nauka, Moscow, 1975; English transl., Grundlehren der Mathematischen Wissenschaften, vol. 263, Springer-Verlag, Berlin, 1984. MR 736839 (85b:47057)

[17] M. A. Krasnosel'skiı̌, The operator of shift along trajectories of differential equations, Nauka, Moscow, 1966. (Russian) MR0203159 (34:3012) 
[18] M. A. Krasnosel'skiı̌, A. I. Perov, A. I. Povolotskiı̌, and P. P. Zabrě̌ko, Vector fields on the plane, Fizmatgiz, Moscow, 1963. (Russian)

[19] A. C. Lazer and P. J. McKenna, Large-amplitude periodic oscillations in suspension bridges: Some new connections with nonlinear analysis, SIAM Rev. 32 (1990), 537-578. MR1084570 (92g:73059)

[20] J. Leray and J. Schauder, Topologie et équations fonctionnelles, Ann. Sci. École Norm. Sup. (3) 51 (1934), 45-78. MR1509338

[21] S. Lefschetz, Differential equations: Geometric theory, Pure and Applied Mathematics, Vol. VI, Interscience (division of John Wiley), New York-London, 1963. MR0094488 (20:1005)

[22] O. Makarenkov and P. Nistri, Periodic solutions for planar autonomous systems with nonsmooth periodic perturbations, J. Math. Anal. Appl. 338 (2008), 1401-1417. MR2386507 (2008m:34099)

[23] O. Yu. Makarenkov, On one approach in the theory of ordinary differential equations with a small parameter, Diploma thesis, Math. Fac., Voronezh Univ., 2003. (Russian)

[24] I. G. Malkin, On Poincaré's theory of periodic solutions, Prikl. Mat. Mekh. 13 (1949), 633-646. (Russian) MR0036384 (12:100j)

[25] J. Mawhin, Le problème des solutions périodiques en mécanique non linéaire, Thèse de doctorat en sciences, Université de Liège, 1969.

[26] J. Mawhin, Degré topologique et solutions périodiques des systèmes différentiels non linéaires, Bull. Soc. Roy. Sci. Liège 38 (1969), 308-398. MR0594965 (58:28863)

[27] V. K. Mel'nikov, On the stability of a center for time-periodic perturbations, Trudy Moskov. Mat. Obshch. 12 (1963), 3-52. (Russian) MR0156048 (27:5981)

[28] Yu. A. Mitropol'skiı̌, The method of averaging in nonlinear mechanics, Naukova Dumka, Kiev, 1971. (Russian) MR0664945 (58:32173)

[29] Yu. A. Mitropol'skiı̌, On periodic solutions of systems of nonlinear differential equations with non-differentiable right-hand sides, Ukrain. Mat. Zh. 11 (1959), 366-379. (Russian) MR0141846 (25:5243)

[30] A. D. Morozov, Degenerate resonances in Hamiltonian systems with $3 / 2$ degress of freedom, Chaos 12 (2002), 539-548. MR.1939451 (2003i:37050)

[31] A. D. Morozov, A complete qualitative investigation of Duffing's equation, Differentsial'nye Uravneniya 12 (1976), 241-255. (Russian) MR0409973 (53:13724)

[32] A. D. Morozov and L. P. Shil'nikov, On nonconservative periodic systems close to two-dimensional Hamiltonian ones, Prikl. Mat. Mekh. 47 (1983), 385-394; English transl., J. Appl. Math. Mech. 47 (1984), 327-334. MR760165 (86c:58052)

[33] F. Nakajima and G. Seifert, The number of periodic solutions of 2-dimensional periodic systems, J. Differential Equations 49 (1983), 430-440. MR715695 (85a:34065)

[34] R. Ortega, A criterion for asymptotic stability based on topological degree, World Congress of Nonlinear Analysts '92 (Tampa, FL, 1992), de Gruyter, Berlin, 1996, pp. 383-394. MR1389089

[35] J. Palis, Jr. and W. de Melo, Geometric theory of dynamical systems. An introduction, SpringerVerlag, New York-Berlin, 1982. MR669541(84a:58004)

[36] O. Perron, Die Ordnungszahlen linearer Differentialgleichungssysteme, Math. Z. 31 (1930), 748 766. MR.1545146

[37] L. S. Pontryagin, Ordinary differential equations, Nauka, Moscow, 1974; English transl. (of a previous edition), Addison-Wesley, Reading, Mass.-Palo Alto, Calif.-London, 1962. MR0140742 $(25: 4156)$

[38] H. Poincaré, Sur les courbes définies par les équations différentielles. I, II, Jordan J. (4) I (1885), 167-244; ibid. II (1886) 151-211.

[39] M. B. H. Rhouma and C. Chicone, On the continuation of periodic orbits, Methods Appl. Anal. 7 (2000), 85-104. MR.1796007 (2001h:34064)

[40] A. M. Samollenko, On periodic solutions of differential equations with nondifferentiable right-hand sides, Ukrain. Mat. Zh. 15 (1963), 328-332. (Russian) MR.0159990 (28:3204)

[41] R. A. Smith, Some applications of Hausdorff dimension inequalities for ordinary differential equations, Proc. Roy. Soc. Edinburgh Sect. A 104 (1986), 235-259. MR877904 (88a:34056)

[42] K. Yagasaki, The Melnikov theory for subharmonics and their bifurcations in forced oscillations, SIAM J. Appl. Math. 56 (1996), 1720-1765. MR1417478(97k:34061)

[43] Y. Zhao, On the monotonicity of the period function of a quadratic system, Discrete Contin. Dyn. Syst. 13 (2005), 795-810. MR2153144 (2006d:34072)

Research Institute of Mathematics, Voronezh State University, Voronezh, Russia E-mail address: omakarenkov@math.vsu.ru 